\title{
Learning Nondeterministic Real-Time Automata
}

\author{
JIE AN, Max Planck Institute for Software Systems, Germany \\ BOHUA ZHAN and NAIJUN ZHAN, SKLCS and Science \& Technology on Integrated Information \\ System Laboratory, Institute of Software, CAS, China and University of Chinese Academy of Sciences, China \\ MIAOMIAO ZHANG, School of Software Engineering, Tongji University, China
}

\begin{abstract}
We present an active learning algorithm named NRTALearning for nondeterministic real-time automata (NRTAs). Real-time automata (RTAs) are a subclass of timed automata with only one clock which resets at each transition. First, we prove the corresponding Myhill-Nerode theorem for real-time languages. Then we show that there exists a unique minimal deterministic real-time automaton (DRTA) recognizing a given real-time language, but the same does not hold for NRTAs. We thus define a special kind of NRTAs, named residual real-time automata (RRTAs), and prove that there exists a minimal RRTA to recognize any given real-time language. This transforms the learning problem of NRTAs to the learning problem of RRTAs. After describing the learning algorithm in detail, we prove its correctness and polynomial complexity. In addition, based on the corresponding Myhill-Nerode theorem, we extend the existing active learning algorithm $N L^{*}$ for nondeterministic finite automata to learn RRTAs. We evaluate and compare the two algorithms on two benchmarks consisting of randomly generated NRTAs and rational regular expressions. The results show that NRTALearning generally performs fewer membership queries and more equivalence queries than the extended $N L^{*}$ algorithm, and the learnt NRTAs have much fewer locations than the corresponding minimal DRTAs. We also conduct a case study using a model of scheduling of final testing of integrated circuits.
\end{abstract}

\section{CCS Concepts: • Computer systems organization $\rightarrow$ Real-time languages; • Theory of computation $\rightarrow$ Regular languages;}

Additional Key Words and Phrases: Active learning, model learning, nondeterministic real-time automata, real-time languages

\section{ACM Reference format:}

Jie An, Bohua Zhan, Naijun Zhan, and Miaomiao Zhang. 2021. Learning Nondeterministic Real-Time Automata. ACM Trans. Embedd. Comput. Syst. 20, 5s, Article 99 (September 2021), 26 pages.

https://doi.org/10.1145/3477030

This article appears as part of the ESWEEK-TECS special issue and was presented in the International Conference on Embedded Software (EMSOFT), 2021.

This work is supported in part by the Deutsche Forschungsgemeinschaft project 389792660-TRR 248, the CAS Pioneer Hundred Talents Program under grant No. Y9RC585036, and the NSFC under grants No. 61625206, 61732001, 61972284, and 62032024 .

Authors' addresses: J. An, Max Planck Institute for Software Systems, Paul-Ehrlich Str. G 26, Kaiserslautern, Germany, 67663; email: jiean@mpi-sws.org; B. Zhan and N. Zhan, SKLCS and Science \& Technology on Integrated Information System Laboratory, Institute of Software, CAS, Beijing, China, 100190 and University of Chinese Academy of Sciences, Beijing, China, 100049; emails: \{bzhan, znj\}@ios.ac.cn; M. Zhang, School of Software Engineering, Tongji University, Shanghai, China, 201804; email: miaomiao@tongji.edu.cn.

Permission to make digital or hard copies of all or part of this work for personal or classroom use is granted without fee provided that copies are not made or distributed for profit or commercial advantage and that copies bear this notice and the full citation on the first page. Copyrights for components of this work owned by others than the author(s) must be honored. Abstracting with credit is permitted. To copy otherwise, or republish, to post on servers or to redistribute to lists, requires prior specific permission and/or a fee. Request permissions from permissions@acm.org.

(c) 2021 Copyright held by the owner/author(s). Publication rights licensed to ACM.

1539-9087/2021/09-ART99 $\$ 15.00$

https://doi.org/10.1145/3477030 


\section{INTRODUCTION}

In recent decades, model learning has attracted increasing attentions in many communities, especially formal methods and artificial intelligence, since it has wide applications in model checking [3], analysis of protocols [17], grammatical inference [11], interpretation of neural networks [34, 35] and so on. For model learning technique, a seminal work is the minimally adequate teacher (MAT) framework [7] proposed by Angluin to learn regular languages in 1987. In the MAT framework, a learner actively learns a regular language from a teacher using membership and equivalence queries. For a membership query, the learner asks whether a word belongs to the target language. For an equivalence query, the learner submits a deterministic finite automaton to the teacher as a hypothesis for the target language. The teacher can answer yes or no for the queries and in the latter case, provide a counterexample as evidence for the differences between the current hypothesis and the target. Following this approach, many efficient algorithms have been proposed for active learning of different kinds of automata. We refer to the survey [27] for a comprehensive introduction.

For real-time and embedded systems, timing constraints play a key role in the correctness and safety of the system. Classical finite automata is unable to describe the infinite number of timed actions. Instead, Timed automata [2], a kind of finite automata extended with a finite number of real-valued clocks, are widely used to model real-time and embedded systems. In this paper, we consider the active learning problem of real-time automata (RTAs), a subclass of timed automata with a single clock that resets at every transition, with the goal to learn nondeterministic RTAs for real-time languages in the MAT framework. RTAs yield simple models while preserving adequate expressiveness, and therefore have been widely used in practical real-time systems, e.g. scheduling of real-time tasks [25] and key-distribution protocols [14]. In [15], Dima introduced real-time automata and revealed some of its important properties, including the Kleene theorem for RTAs, which shows that the expressiveness of rational regular expressions, deterministic realtime automata (DRTAs) and nondeterministic real-time automata (NRTAs) are all equivalent. On the other hand, the Kleene theorem also tells us that a DRTA can be exponentially bigger than an equivalent NRTA in terms of the number of locations, which is similar to the situation of DFAs and NFAs. Hence, compared to learning DRTAs, learning NRTAs may lead to more succinct models, which will be more useful, especially for applications in verification.

The cornerstone of learning regular languages in the MAT framework is the Myhill-Nerode theorem which shows that a language $L$ is regular if and only if the right-congruence relation $R_{L}$ has a finite number of equivalence classes. Thus, we can map each equivalence class to a location (state) in a finite automaton, and this implies that there is a unique minimal DFA which recognizes $L$. However, there is no unique minimal NFA for the language, which means it is not clear which target automaton should be learned in the MAT framework. Hence, Bollig et al. proposed an algorithm named $N L^{*}$ [8] to learn a subclass of NFAs named residual finite state automata (RFSAs) [12], which have the property that there is a unique minimal RFSA recognizing a given regular language. Inspired by their work, we first prove a corresponding Myhill-Nerode theorem for real-time languages which shows that there exists a unique minimal DRTA for a given realtime language. However, there is no unique minimal NRTA, so we proceed to define the notions of residual real-time languages and residual real-time automata (RRTAs). By proving that there exists a unique minimal RRTA for a given real-time language, we transform the learning problem of NRTAs to the learning problem of RRTAs. Compared to $N L^{*}$, the challenge is to handle nondeterministic behaviours caused by timing information in timed words instead of untimed actions. By carefully designing the readiness conditions of the observation table and the process for handling counterexamples, we present an active learning algorithm named NRTALearning. Based on 
the corresponding Myhill-Nerode theorem, we can also directly extend $N L^{*}$ to learn RRTAs. We prove the correctness and termination of both algorithms, and show the polynomial complexity of both algorithms in terms of the number of queries. The two algorithms have been implemented and evaluated on two benchmarks consisting of randomly generated NRTAs and rational regular expressions. The results show that the algorithm NRTALearning generally performs fewer membership queries and more equivalence queries than the extended $N L^{*}$ algorithm. Additionally, the learnt NRTAs have much fewer locations than the corresponding minimal DRTAs. Finally, we show a case study using a model of scheduling of final testing of integrated circuits.

In summary, our main contributions are as follows.

- A version of Myhill-Nerode theorem for real-time languages.

- Definition of residual real-time automata, and a proof that there is a unique minimal RRTA recognizing a given real-time language.

- Two efficient active learning algorithms for NRTAs. One is in the standard MAT framework, and the other needs an assumption.

- Implementation and experimental evaluation on two benchmarks for learning NRTAs and rational regular expressions. ${ }^{1}$

Related work. There are several works on learning timed models. We first introduce works in the active learning paradigm. In [18], Grinchtein et al. proposed a learning algorithm for deterministic event-recording automata (ERAs) which are a kind of timed automata that, for every untimed action $a$, a clock is used to record the time length from the last occurrence of $a$ to now. In [19], Henry et al. considered learning a kind of deterministic ERAs, named reset-free ERAs. However, Dima pointed out that RTAs are incomparable to ERAs since RTA may accept languages consisting of two actions separated by an interval with integer length while ERAs may not [15]. An et al. proposed an active learning algorithm for deterministic one-clock timed automata in [4]. However, guessing reset information of the clock leads to a combinatorial explosion in the number of candidate tables, and thus an exponential complexity. Hence, they considered learning DRTAs with a similar technique [5]. In this paper, we consider learning NRTAs directly. Another kind of simple timed model is called Mealy machine with timers. The value of each timer decreases and a timeout is triggered when the value becomes 0 . Caldwell et al. proposed an algorithm on learning such a model from programmable logic controllers [10]. In [28], Vaandrager et al. presented an efficient learning algorithm for such models with one timer. Passive learning for timed models has also attracted much interest. Passive learning aims at identifying a model from a given data set and the learnt model is only required to be consistent with the data set. Based on the classic identification method for DFAs, named evidence-driven state-merging (EDSM), Verwer et al. proposed the RTI algorithm for identifying DRTAs in the limit $[32,33]$. After that, they presented a passive learning algorithm for deterministic one-clock timed automata [29-31]. Additionally, the passive learning methods cited above concern only discrete-time semantics of the timed models, i.e., the clock valuations are non-negative integers. There are also some works incorporating other techniques from machine learning, e.g., learning deterministic timed automata via genetic programming (GP) [26] and learning probabilistic real-time automata via clustering techniques [23]. Recently, Aichernig et al. extended their GP-based learning method in an active manner using conformance testing [1] and successfully learned models with large size. The conformance relation requires that the learnt deterministic model and the system under test agree on a finite set of sampled traces. Thus it cannot guarantee the correctness of the learnt model. Even such techniques have been applied to

\footnotetext{
${ }^{1}$ The implementation and the experiments are available at https://github.com/Leslieaj/NRTALearning.
} 
learn hybrid automata [20, 24]. To the best of our knowledge, our paper is the first work on active learning of a kind of nondeterministic timed automata.

Organization. In the following, Section 2 recalls important preliminary definitions. The corresponding Myhill-Nerode Theorem for real-time languages is presented and proved in Section 3. We then define residual real-time languages and residual real-time automata in Section 4. Two active learning algorithms for NRTAs are proposed in Section 5 and evaluated in Section 6. Finally, we conclude the paper in Section 7.

\section{PRELIMINARIES}

In this section, we recall some notions including timed words, timed automata, real-time automata and their recognized timed languages. Let $\mathbb{R}_{\geq 0}$ and $\mathbb{N}$ be the set of non-negative real numbers and natural numbers, respectively, and $\mathbb{B}$ the Boolean set. We use $T$ to stand for true and $\perp$ for false Let $\Sigma$ be a set of actions considered in this paper.

A (delay) timed word over $\Sigma \times \mathbb{R}_{\geq 0}$ is a finite sequence $\omega=\left(\sigma_{1}, \tau_{1}\right)\left(\sigma_{2}, \tau_{2}\right) \cdots\left(\sigma_{n}, \tau_{n}\right)$, where $\sigma_{i} \in \Sigma$ and $\tau_{i} \in \mathbb{R}_{\geq 0}$ for $1 \leq i \leq n,|\omega|=n$ is the length of $\omega$, and each $\tau_{i}$ represents the delay time between two consecutive actions. We use $\epsilon$ to represent the empty word with $|\epsilon|=0$. A timed word $\omega$ is called a timed action if $|\omega|=1$. A timed language $\mathcal{L}$ can be viewed as a set of timed words.

Timed automata [2], an extension of finite automata with a finite number of real-valued clocks, are widely used to model real-time systems. Let $C$ be the set of clock variables, and let $\Phi_{C}$ be the set of clock constraints of the form $\phi::=\top|c \bowtie m| \phi \wedge \phi$, where $c \in C, m \in \mathbb{N}$ and $\bowtie \in\{=,<,>, \leq, \geq\}$. A clock valuation is a function $v: C \rightarrow \mathbb{R}_{\geq 0}$ that assigns a non-negative real number to the clocks. For $t \in \mathbb{R}_{\geq 0}$, let $v+t$ be the clock valuation with $(v+t)(c)=v(c)+t$ for all $c \in C$.

In this paper, we consider a subclass of timed automata with a single clock which resets at every transition, termed real-time automata [15]. According to the definitions of clock constraint and clock valuation, a transition guard in a real-time automaton can be represented by an interval with endpoints in $\mathbb{N} \cup\{\infty\}$. For example, let $c$ be the unique clock, $\phi_{1}: c<5 \wedge c \geq 3$ is represented as $[3,5), \phi_{2}: c=6$ as $[6,6]$, and $\phi_{3}: \top$ as $[0, \infty)$. We omit the single clock and give a more succinct definition as follows.

Definition 2.1 (Nondeterministic Real-time Automata). A (nondeterministic) real-time automaton is a tuple $\mathcal{A}=\left(Q, \Sigma, \Delta, Q_{0}, F\right)$ where

- $Q$ is a finite set of locations;

- $\Sigma$ is a finite alphabet;

- $\Delta \subseteq Q \times \Sigma \times 2^{\mathbb{R}_{\geq 0}} \times Q$ is a transition relation with $|\Delta|<\infty$, where $2^{\mathbb{R}_{\geq 0}}$ represents the set of intervals whose endpoints are in $\mathbb{N} \cup\{\infty\}$;

- $Q_{0} \subseteq Q$ is a finite set of initial locations;

- $F \subseteq Q$ is a finite set of accepting locations.

A transition $\left(q, \sigma, I, q^{\prime}\right) \in \Delta$ allows a jump from the source location $q$ to the target location $q^{\prime}$ by performing the action $\sigma \in \sum$ if the guard $I$ is satisfied (i.e., $v(c) \in I$ ). Meanwhile, clock $c$ is reset to zero. Since the unique clock $c$ resets at every transition, the value of the logic clock $c$ represents the delay time between two actions. Thus, $\Delta$ induces the transition function $\delta: Q \times \Sigma \times \mathbb{R}_{\geq 0} \rightarrow 2^{Q}$ such that $\delta(q,(\sigma, \tau))=\left\{q^{\prime} \in Q \mid\left(q, \sigma, I, q^{\prime}\right) \in \Delta, \sigma \in \Sigma, v(c)=\tau \in I\right\}$. We extend $\delta$ to $\delta: Q \times(\Sigma \times$ $\left.\mathbb{R}_{\geq 0}\right)^{*} \rightarrow 2^{Q}$ by $\delta(q, \epsilon)=\{q\}$ and $\delta(q,(\sigma, \tau) \cdot \omega)=\bigcup_{q^{\prime} \in \delta(q,(\sigma, \tau))} \delta\left(q^{\prime}, \omega\right)$, and subsequently to the set of locations $Q^{\prime} \subseteq Q$ by $\delta\left(Q^{\prime}, \omega\right)=\bigcup_{q \in Q^{\prime}} \delta(q, \omega)$. 


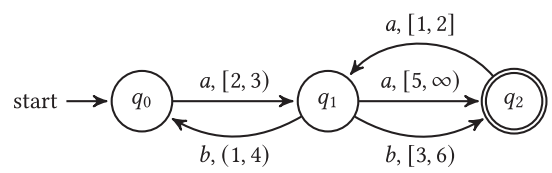

Fig. 1. An NRTA $\mathcal{A}$ with a set $Q_{0}=\left\{q_{0}\right\}$ of initial locations and a set $F=\left\{q_{2}\right\}$ of accepting locations.

A run of an RTA $\mathcal{A}$ is either a single initial state $\rho=q_{0} \in Q_{0}$ or a finite sequence $\rho=q_{0} \underset{\tau_{1}}{\stackrel{\sigma_{1}}{\longrightarrow}}$ $q_{1} \underset{\tau_{2}}{\stackrel{\sigma_{2}}{\longrightarrow}} \cdots \underset{\tau_{n}}{\stackrel{\sigma_{n}}{\longrightarrow}} q_{n}$, with $n>0,\left(q_{i-1}, \sigma_{i}, I_{i}, q_{i}\right) \in \Delta$, and $\tau_{i} \in I_{i}$ for $1 \leq i \leq n$. When action $\sigma_{i}$ is being performed, $v(c)=\tau_{i}$. After that, the clock $c$ resets to 0 .

The trace of a run $\rho$ is a timed word defined as: $\operatorname{trace}\left(q_{0}\right)=\epsilon$, and if $\rho=q_{0} \underset{\tau_{1}}{\stackrel{\sigma_{1}}{\longrightarrow}} q_{1} \underset{\tau_{2}}{\stackrel{\sigma_{2}}{\longrightarrow}} \cdots \underset{\tau_{n}}{\longrightarrow} q_{n}$ then trace $(\rho)=\left(\sigma_{1}, \tau_{1}\right)\left(\sigma_{2}, \tau_{2}\right) \cdots\left(\sigma_{n}, \tau_{n}\right)$. For an RTA $\mathcal{A}$, its recognized timed language can be defined on traces as $\mathcal{L}(\mathcal{A})=\left\{\operatorname{trace}(\rho) \mid \rho\right.$ starts from $q_{0} \in Q_{0}$ and ends in $\left.q_{n} \in F\right\}$. Given an RTA, $\mathcal{L}_{q}$ denotes the timed language starting from a location $q \in Q$, which is the set of timed words $\omega$ such that $\delta(q, \omega) \cap F \neq \emptyset$. In this paper, we consider the real-time languages defined as follows.

Definition 2.2 (Real-time Languages). Given a timed language $\mathcal{L} \subseteq\left(\Sigma \times \mathbb{R}_{\geq 0}\right)^{*}, \mathcal{L}$ is a real-time language if $\mathcal{L}$ can be recognized by an RTA $\mathcal{A}$, i.e. $\mathcal{L}=\mathcal{L}(\mathcal{A})$.

An RTA is a deterministic real-time automaton (DRTA) if and only if there is at most one run for a given timed word $\omega$, i.e. $\left|Q_{0}\right|=1$ and $|\delta(q,(\sigma, \tau))|=1$ for all $q \in Q$ and $(\sigma, \tau) \in \Sigma \times \mathbb{R}_{\geq 0}$. Otherwise, we call it nondeterministic real-time automaton (NRTA). Therefore, given a timed word $\omega$, it is accepted by an NRTA $\mathcal{A}$ if and only if one of its corresponding runs ends in an accepting location $q \in F$ of $\mathcal{A}$. According to the Kleene Theorem for RTAs [15], DRTAs and NRTAs have the same expressiveness. And thus every real-time language in our definition is regular.

Example 2.3. Consider NRTA $\mathcal{A}=\left(Q, \Sigma, \Delta, Q_{0}, F\right)$ in Figure 1 . The set of locations $Q=$ $\left\{q_{0}, q_{1}, q_{2}\right\}$, the finite alphabet $\Sigma=\{a, b\}$, the set of initial locations $Q_{0}=\left\{q_{0}\right\}$, the set of accepting locations $F=\left\{q_{2}\right\}$, and $\Delta=\left\{\left(q_{0}, a,[2,3), q_{1}\right),\left(q_{1}, a,[5, \infty), q_{2}\right),\left(q_{1}, b,(1,4), q_{0}\right),\left(q_{1}, b,[3,6), q_{2}\right)\right.$, $\left.\left(q_{2}, a,[1,2], q_{1}\right)\right\}$. For the timed word $\omega=(a, 2.1)(b, 3)$, there are two runs in $\mathcal{A}$, i.e., $\rho_{1}=q_{0} \underset{2.1}{\stackrel{a}{\longrightarrow}}$ $q_{1} \underset{3}{\stackrel{b}{\rightarrow}} q_{0}$ and $\rho_{2}=q_{0} \underset{2.1}{\stackrel{a}{\longrightarrow}} q_{1} \underset{3}{\stackrel{b}{\rightarrow}} q_{2}$, corresponding to it. Clearly, $\omega$ is accepted by NRTA $\mathcal{A}$ as $\rho_{2}$ ends in an accepting location $q_{2} \in F$.

\section{THE MYHILL-NERODE THEOREM FOR REAL-TIME LANGUAGES}

In order to prove the Myhill-Nerode Theorem for real-time languages, we first recall the notion of the region [2]. Since there is only one clock $c$, given a clock valuation $v$, we define the region $\llbracket v \rrbracket$ containing $v$ as $\llbracket v \rrbracket=[v, v]$ if $v \in \mathbb{N}$, and $\llbracket v \rrbracket=(\lfloor v\rfloor,\lfloor v\rfloor+1)$ otherwise, where $\lfloor v\rfloor$ is the integer part of $v$. As a convention, $\llbracket v \rrbracket=(\kappa, \infty)$ if $v$ is greater than the maximum constant $\kappa$ appearing in the RTA. Hence, there exist $2 \kappa+2$ such regions, including $[n, n]$ with $0 \leq n \leq \kappa,(n, n+1)$ with $0 \leq n<\kappa$, and $(\kappa, \infty)$. We further define the region words as follows.

Definition 3.1 (Region Words). Given a timed word $\omega=\left(\sigma_{1}, \tau_{1}\right)\left(\sigma_{2}, \tau_{2}\right) \cdots\left(\sigma_{n}, \tau_{n}\right)$, a word $\gamma=$ $\left(\sigma_{1}, \llbracket \tau_{1} \rrbracket\right)\left(\sigma_{1}, \llbracket \tau_{2} \rrbracket\right) \cdots\left(\sigma_{n}, \llbracket \tau_{n} \rrbracket\right)$ is the region word of $\omega$, denoted as $\gamma=\llbracket \omega \rrbracket$.

The key concept in the classic Myhill-Nerode Theorem is that of indistinguishable words (the right-congruence relation). Hence, we introduce two definitions about indistinguishable timed words as follows. 
Definition 3.2. Let $\mathcal{L} \subseteq\left(\Sigma \times \mathbb{R}_{\geq 0}\right)^{*}$ be any timed language. Two timed words $\omega_{1}, \omega_{2} \in(\Sigma \times$ $\left.\mathbb{R}_{\geq 0}\right)^{*}$ are indistinguishable by $\mathcal{L}$, denoted $\omega_{1} \sim \mathcal{L} \omega_{2}$, if for every timed word $\omega^{\prime} \in\left(\Sigma \times \mathbb{R}_{\geq 0}\right)^{*}$, it holds that $\omega_{1} \cdot \omega^{\prime} \in \mathcal{L}$ if and only if $\omega_{2} \cdot \omega^{\prime} \in \mathcal{L}$.

Definition 3.3. Given a DRTA $\mathcal{A}$, two timed words $\omega_{1}, \omega_{2} \in\left(\Sigma \times \mathbb{R}_{\geq 0}\right)^{*}$ are indistinguishable by $\mathcal{A}$, denoted $\omega_{1} \sim \mathcal{A} \omega_{2}$, if $\delta\left(q_{0}, \omega_{1}\right)=\delta\left(q_{0}, \omega_{2}\right)$, i.e. the reachable location for $\omega_{1}$ is the same as the reachable location for $\omega_{2}$.

LEMmA 3.4. Given a DRTA $\mathcal{A}$, for all $\omega_{1}, \omega_{2} \in\left(\Sigma \times \mathbb{R}_{\geq 0}\right)^{*}, \omega_{1} \sim \mathcal{A} \omega_{2}$ if $\omega_{1}$ and $\omega_{2}$ belong to the same region word $\gamma$.

LEMmA 3.5. If a timed language $\mathcal{L}=\mathcal{L}(\mathcal{A})$ for a DRTA $\mathcal{A}$, then for all $\omega_{1}, \omega_{2} \in\left(\Sigma \times \mathbb{R}_{\geq 0}\right)^{*}$, if $\omega_{1} \sim_{\mathcal{A}} \omega_{2}$ then $\omega_{1} \sim_{\mathcal{L}} \omega_{2}$.

COROLLARY 3.6. If $\mathcal{L}$ is a real-time language, then $\sim_{\mathcal{L}}$ has a finite number of equivalence classes.

It's not hard to prove the above corollary and lemmas. Based on them, we claim that there is a corresponding Myhill-Nerode theorem for real-time languages as follows.

Theorem 3.7 (MyHill-Nerode theOrem For REAL-Time languages). $\mathcal{L}$ is a real-time language if and only if $\sim_{\mathcal{L}}$ has a finite number of equivalence classes which satisfy the following two conditions:

1. For all $\omega \in\left(\Sigma \times \mathbb{R}_{\geq 0}\right)^{*}, \sigma \in \Sigma$ and $\tau, \tau^{\prime} \in \mathbb{R}_{\geq 0}$, if $\llbracket \tau^{\prime} \rrbracket=\llbracket \tau \rrbracket$, then $\omega \cdot\left(\sigma, \tau^{\prime}\right) \sim_{\mathcal{L}} \omega \cdot(\sigma, \tau)$;

2. There exists $\kappa \in \mathbb{N}$, such that for all $\sigma \in \Sigma$ and $\tau, \tau^{\prime} \in \mathbb{R}_{\geq 0}$, if $\tau>\kappa$ and $\tau^{\prime}>\kappa$ then $\omega \cdot(\sigma, \tau) \sim{ }_{\mathcal{L}} \omega \cdot\left(\sigma, \tau^{\prime}\right)$.

Furthermore there is a unique minimal (w.r.t. the number of locations) DRTA $\mathcal{A}$ with $\mathcal{L}(\mathcal{A})=\mathcal{L}$.

Proof. Corollary 3.6 shows that if $\mathcal{L}$ is a real-time language, then $\sim_{\mathcal{L}}$ has finitely many equivalence classes. We further prove that $\sim \mathcal{L}$ satisfies the above two conditions. Suppose that a DRTA $\mathcal{A}$ recognizes the real-time language $\mathcal{L}$. For the first condition, we assume that $\mathcal{A}$ ends in a location $q$ after reading $\omega \cdot(\sigma, \tau)$. By LemMA 3.4, for all $\tau^{\prime} \in \llbracket \tau \rrbracket$, we have $\omega \cdot\left(\sigma, \tau^{\prime}\right) \sim_{\mathcal{A}} \omega \cdot(\sigma, \tau)$. Then by Lemma 3.5, we have $\omega \cdot\left(\sigma, \tau^{\prime}\right) \sim \mathcal{L} \omega \cdot(\sigma, \tau)$. For the second condition, we let $\kappa$ be the maximum constant appearing in the timed constraints of $\mathcal{A}$. By the definitions of DRTAs and regions, if $\tau>\kappa$ and $\tau^{\prime}>\kappa$, then $\llbracket \tau \rrbracket=\llbracket \tau^{\prime} \rrbracket=(\kappa, \infty)$. By LemmA 3.4, $\omega \cdot(\sigma, \tau) \sim_{\mathcal{A}} \omega \cdot\left(\sigma, \tau^{\prime}\right)$. Then by Lemma $3.5, \omega \cdot(\sigma, \tau) \sim \mathcal{L} \omega \cdot\left(\sigma, \tau^{\prime}\right)$.

Then we prove the other direction. If $\sim_{\mathcal{L}}$ has finitely many equivalence classes which satisfy the two conditions, we can build a DRTA $\mathcal{A}=\left(Q, \Sigma, \Delta, Q_{0}, F\right)$ which recognizes $\mathcal{L}$ as follows. Let $\mathbb{L}_{0}, \mathbb{L}_{1}, \ldots, \mathbb{L}_{n}$ be the disjoint equivalence classes of $\sim_{\mathcal{L}}$, such that $\epsilon \in \mathbb{L}_{0}$. Note that the union of the equivalence classes is $\left(\Sigma \times \mathbb{R}_{\geq 0}\right)^{*}$. First, we build the set of locations. We set $Q=\left\{q_{0}, q_{1}, \ldots, q_{n}\right\}$, where each $q_{i}$ represents the equivalence class $\mathbb{L}_{i}$. Then for building a transition $\left(q_{i}, \sigma, \llbracket \tau \rrbracket, q_{j}\right)$, where $\sigma \in \Sigma, \tau \in \mathbb{R}_{\geq 0}$ and $0 \leq i, j \leq n$, we select a timed word $\omega$ from $\mathbb{L}_{i}$ and then find $q_{j}$ such that $\omega \cdot(\sigma, \tau) \in \mathbb{L}_{j}$. Since $\sim_{\mathcal{L}}$ satisfies the two conditions, the number of regions $\llbracket \tau \rrbracket$ is finite. Therefore, $\Delta$ is a finite set. Finally, the initial location is $q_{0}$ and the set of accepting locations is $F=\left\{q_{i} \mid \mathbb{L}_{i} \subseteq \mathcal{L}\right\}$. By induction, we can prove $\delta\left(q_{0}, \omega\right)=q_{i} \in F \Leftrightarrow \omega \in \mathbb{L}_{i} \subseteq \mathcal{L}$, i.e. $\mathcal{L}(\mathcal{A})=\mathcal{L}$.

Combining the two directions, $\mathcal{L}$ is a real-time language if and only if $\sim_{\mathcal{L}}$ has finitely many equivalence classes. Furthermore, we can build a unique minimal DRTA $\mathcal{A}$ with $\mathcal{L}(\mathcal{A})=\mathcal{L}$.

\section{RESIDUAL REAL-TIME AUTOMATA}

From TheOREM 3.7, we know that there exists a unique minimal DRTA which can recognize a given real-time language. However, the same does not hold for NRTAs, which means that there is no unique minimal NRTA as the learning target for a given real-time language. Therefore, inspired by Denis et al.'s work on residual finite state automata (RFSAs) [12], we introduce a special 
kind of NRTAs, named residual real-time automata (RRTAs), which have nice properties for active learning, that is for a real-time language there is a unique minimal RRTA recognizing it. Then we can transform the problem of actively learning NRTAs to the problem of actively learning RRTAs, which will be the focus of Section 5 .

Definition 4.1 (Residual Real-time Language). Let $\mathcal{L} \subseteq\left(\Sigma \times \mathbb{R}_{\geq 0}\right)^{*}$ be a real-time language and $\omega$ be a timed word. The residual real-time language of $\mathcal{L}$ with regard to $\omega$ is defined by $\omega^{-1} \mathcal{L}=$ $\left\{\omega^{\prime} \in\left(\Sigma \times \mathbb{R}_{\geq 0}\right)^{*} \mid \omega \omega^{\prime} \in \mathcal{L}\right\}$. Let $\operatorname{Res}(\mathcal{L})$ be the set of residual real-time languages of $\mathcal{L}$.

If $\mathcal{L}$ is recognized by an NRTA $\mathcal{A}=\left(Q, \Sigma, \Delta, Q_{0}, F\right)$, then $q \in \delta\left(Q_{0}, \omega\right) \Rightarrow \mathcal{L}_{q} \subseteq \omega^{-1} \mathcal{L}$. Based on Theorem 3.7 and the definition of residual real-time language, if RTA $\mathcal{A}^{\prime}=\left(Q^{\prime}, \Sigma, \Delta^{\prime}, Q_{0}^{\prime}, F^{\prime}\right)$ is the unique minimal DRTA recognizing $\mathcal{L}$, then (1) there exists a unique $q^{\prime} \in Q^{\prime}$ such that $\mathcal{L}_{q^{\prime}}=\omega^{-1} \mathcal{L}$ for each $\omega^{-1} \mathcal{L} \neq \emptyset$; (2) there exists a unique residual language $\omega^{-1} \mathcal{L}$ such that $\omega^{-1} \mathcal{L}=\mathcal{L}_{q^{\prime}}$ for each $q^{\prime} \in Q^{\prime}$. In other words, for the minimal DRTA $\mathcal{A}^{\prime}$, there is a bijection between the set of locations $Q^{\prime}$ and the set of residual real-time languages $\operatorname{Res}\left(\mathcal{L}\left(\mathcal{A}^{\prime}\right)\right)$ (i.e. $\operatorname{Res}(\mathcal{L})$ ).

Definition 4.2 (Residual Real-time Automata). A residual real-time automaton is an NRTA $\mathcal{A}=$ $\left(Q, \Sigma, \Delta, Q_{0}, F\right)$ such that $\forall q \in Q, \exists \omega \in\left(\Sigma \times \mathbb{R}_{\geq 0}\right)^{*}: \mathcal{L}_{q}=\omega^{-1} \mathcal{L}(\mathcal{A})$. Additionally, $\omega$ is called a characterizing timed word for $q$.

Immediately, we can see that a location $q$ in an RRTA $\mathcal{A}$ represents a residual real-time language of $\mathcal{L}(\mathcal{A})$, but not every residual real-time language of $\mathcal{L}(\mathcal{A})$ corresponds to a single location. Suppose $\mathcal{A}^{\prime}$ is the unique minimal DRTA for $\mathcal{L}(\mathcal{A})$, i.e. $\mathcal{L}\left(\mathcal{A}^{\prime}\right)=\mathcal{L}(\mathcal{A})$, since there is a bijection between the set of locations of $\mathcal{A}^{\prime}$ and the set of residual real-time languages of $\mathcal{L}\left(\mathcal{A}^{\prime}\right)$, the locations of RRTA $\mathcal{A}$ are a subset of the locations of the unique minimal DRTA $\mathcal{A}^{\prime}$.

Definition 4.3 (Prime and Composed Residual Real-time Languages). Let $\mathcal{L}$ be a real-time language. A residual real-time language $\omega^{-1} \mathcal{L}$ is called prime if

$$
\bigcup\left\{\omega^{\prime-1} \mathcal{L} \mid \omega^{\prime-1} \mathcal{L} \subsetneq \omega^{-1} \mathcal{L}\right\} \subsetneq \omega^{-1} \mathcal{L},
$$

otherwise, $\omega^{-1} \mathcal{L}$ is called composed.

In other words, $\omega^{-1} \mathcal{L}$ is composed if there exist $\mathcal{L}_{1}, \mathcal{L}_{2}, \ldots, \mathcal{L}_{n} \in \operatorname{Res}(\mathcal{L}) \backslash\left\{\omega^{-1} \mathcal{L}\right\}$ such that $\omega^{-1} \mathcal{L}=\mathcal{L}_{1} \cup \mathcal{L}_{2} \cup \cdots \cup \mathcal{L}_{n}$. Otherwise, it is prime. Additionally, the set of prime residual realtime languages of $\mathcal{L}$ is finite.

The following lemma shows that given an RRTA $\mathcal{A}$, each prime residual language $\omega^{-1} \mathcal{L}(\mathcal{A})$ corresponds to a location of $\mathcal{A}$. In other words, RRTA $\mathcal{A}$ has at least as many locations as the number of the prime residual real-time languages of $\mathcal{L}(\mathcal{A})$.

Lemma 4.4. If $\mathcal{A}=\left(Q, \Sigma, \Delta, Q_{0}, F\right)$ is an RRTA, then there exists a location $q \in Q$ such that $\mathcal{L}_{q}=\omega^{-1} \mathcal{L}(\mathcal{A})$ for each prime residual real-time language $\omega^{-1} \mathcal{L}(\mathcal{A})$.

Proof. (Sketch) Given a prime residual $\omega^{-1} \mathcal{L}(\mathcal{A})$, suppose that $\delta\left(Q_{0}, \omega\right)=\left\{q_{1}, q_{2}, \ldots, q_{m}\right\}$ and let $\omega_{1}, \omega_{2}, \ldots, \omega_{m}$ be the characterizing timed words such that $\mathcal{L}_{q_{i}}=\omega_{i}{ }^{-1} \mathcal{L}(\mathcal{A})$, where $1 \leq i \leq$ $m$. Depending on Definition 4.1, $\omega^{-1} \mathcal{L}(\mathcal{A})=\bigcup_{i=1}^{m} \omega_{i}^{-1} \mathcal{L}(\mathcal{A})$. As $\omega^{-1} \mathcal{L}(\mathcal{A})$ is a prime residual real-time language, according to Definition 4.3, there should exist a $\omega_{i} \in\left\{\omega_{1}, \omega_{2}, \ldots, \omega_{m}\right\}$ such that $\omega_{i}{ }^{-1} \mathcal{L}(\mathcal{A})=\omega^{-1} \mathcal{L}(\mathcal{A})$. Then we find a location $q=q_{i} \in Q$ such that $\mathcal{L}_{q_{i}}=\omega^{-1} \mathcal{L}(\mathcal{A})$.

Definition 4.5 (Canonical Residual Real-time Automata). Let $\mathcal{L}$ be a real-time language. An RRTA $\mathcal{A}=\left(Q, \Sigma, \Delta, Q_{0}, F\right)$ is the canonical residual real-time automaton (CRRTA) of $\mathcal{L}$, where

- $Q=\left\{\omega^{-1} \mathcal{L} \mid \omega^{-1} \mathcal{L}\right.$ is prime $\}$ is the finite set of locations;

- $\sum$ is the alphabet; 
- $\Delta=\left\{\left(\omega_{1}{ }^{-1} \mathcal{L}, \sigma, \llbracket \tau \rrbracket, \omega_{2}{ }^{-1} \mathcal{L}\right) \mid \sigma \in \Sigma \wedge \tau \in \mathbb{R}_{\geq 0} \wedge \omega_{1}{ }^{-1} \mathcal{L}, \omega_{2}{ }^{-1} \mathcal{L} \in Q \wedge \omega_{2}{ }^{-1} \mathcal{L} \subseteq\left(\omega_{1}\right.\right.$. $\left.(\sigma, \tau))^{-1} \mathcal{L}\right\}$ is the transition relation;

- $Q_{0}=\left\{\omega^{-1} \mathcal{L} \mid \omega^{-1} \mathcal{L} \subseteq \mathcal{L} \wedge \omega^{-1} \mathcal{L} \in Q\right\}$ is the set of initial locations;

- $F=\left\{\omega^{-1} \mathcal{L} \mid \epsilon \in \omega^{-1} \mathcal{L} \wedge \omega^{-1} \mathcal{L} \in Q\right\}$ is the set of accepting locations.

According to Theorem 3.7, there exists $\kappa \in \mathbb{N}$ as the maximum value appearing in the timed constraints. Therefore $\Delta$ is finite and the CRRTA of $\mathcal{L}$ is well-defined. The CRRTA is the RRTA with the minimum number of locations (reduced) and maximum number of transitions (saturated).

Theorem 4.6. The canonical residual real-time automaton $\mathcal{A}$ of a real-time language $\mathcal{L}$ is the minimal (w.r.t. the number of locations) RRTA which recognizes $\mathcal{L}$.

Proof. (sketch) It's not hard to prove that the CRRTA $\mathcal{A}$ is an RRTA. Then LEMmA 4.4 shows that it has a minimal number of locations.

Note that the CRRTA $\mathcal{A}$ has a lot of transitions, according to Definition 4.5. One way to reduce the number of transitions is to merge those with the same source location, target location and action, and whose region guards are adjacent. For example, if $\left(q, \sigma,(1,2), q^{\prime}\right)$ and $\left(q, \sigma,[2,2], q^{\prime}\right)$ are two transitions in $\Delta$, then we can merge them into a new transition $\left(q, \sigma,(1,2], q^{\prime}\right)$. Such operations do not change the number of locations and the recognized real-time language of $\mathcal{A}$. If $\mathcal{A}^{\prime}$ is the automaton transformed from a CRRTA $\mathcal{A}$ through the above operation, $\mathcal{A}^{\prime}$ is seen as the CRRTA for the same real-time language hereafter.

\section{LEARNING RESIDUAL REAL-TIME AUTOMATA}

Based on the canonical property of RRTAs, we can transform the problem of actively learning NRTAs to the problem of actively learning minimal RRTAs. In this section, we describe the learning algorithm, analyse its complexity and prove its correctness.

We first describe the settings for active learning of real-time languages in general. Following Angluin's MAT framework, there exists a teacher who knows the target real-time language $\mathcal{L}$ and answers two kinds of queries from a learner. For a membership query, the learner asks whether a timed word $\omega$ is in the language $\mathcal{L}$ or not. The teacher can answer yes or no. The learner collects the results of membership queries in an observation table. For an equivalence query, the teacher receives an NRTA $\mathcal{A}$ from the learner as a hypothesis for $\mathcal{L}$. The teacher answers whether $\mathcal{L}(\mathcal{A})=$ $\mathcal{L}$. If not, the teacher returns a timed word as a counterexample which distinguishes $\mathcal{L}(\mathcal{A})$ and $\mathcal{L}$. In what follows, we present the details of the learning algorithm.

\subsection{Membership Query and Real-time Observation Table}

In order to gather enough information to construct a hypothesis, the learner makes membership queries like "Is the timed word $\omega$ in $\mathcal{L}$ ?". In practice, a membership query is often conducted by testing. In theory, we assume that the teacher has an oracle to answer membership queries, i.e., MQ : $\left(\sum \times \mathbb{R}_{\geq 0}\right)^{*} \rightarrow\{+,-\}$. Given an RTA recognizing $\mathcal{L}$, the teacher gives a positive answer if there is a run $\rho$ ending in an accepting location after reading $\omega$. The results of membership queries are collected in a real-time observation table $\mathcal{T}$ as follows.

Definition 5.1 (Real-time Observation Table). A real-time observation table is a 6-tuple $\mathcal{T}=$ $(\Sigma, \Xi, S, R, E, f)$, where $\Sigma$ is the alphabet, $\Xi=\Sigma \times \mathbb{R}_{\geq 0}$ is the set of all timed actions, $S, R, E \subseteq \Xi^{*}$, $S$ is called the set of prefixes, $R$ the set of extended prefixes and $E$ the set of suffixes, respectively. Specifically,

- $S$ and $R$ are disjoint, i.e. $S \cap R=\emptyset$;

- $S \cup R$ is prefix-closed and $E$ is suffix-closed; 


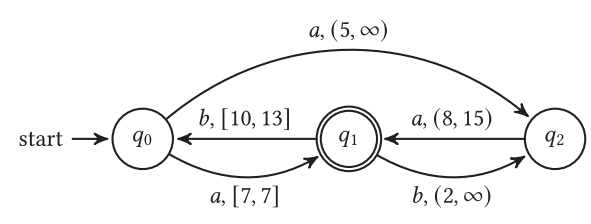

$\mathcal{A}$

\begin{tabular}{r|cccc|c}
\hline $\mathcal{T}$ & $\epsilon$ & $(a, 8.1)$ & $(a, 8.1)(a, 8.1)$ & $(a, 15)$ & \\
\hline$\epsilon$ & - & - & + & - & $\checkmark$ \\
$(a, 5.1)$ & - & + & - & - & $\checkmark$ \\
$(a, 0)$ & - & - & - & - & $\checkmark$ \\
$(a, 5.1)(a, 8.1)$ & + & - & - & - & $\checkmark$ \\
\hline$(b, 0)$ & - & - & - & - & $\checkmark$ \\
$(a, 5.1)(a, 0)$ & - & - & - & - & $\checkmark$ \\
$(a, 5.1)(b, 0)$ & - & - & - & - & $\checkmark$ \\
$\ldots$ & & & $\ldots$ & & $\ldots$ \\
$(a, 7)$ & + & + & - & - & \\
$\ldots$ & & & $\ldots$ & & $\ldots$ \\
\hline
\end{tabular}

Fig. 2. Left: an example of an NRTA. Right: an example of real-time observation table in which the prime prefixes are labelled by $\checkmark$. Some rows of the table are omitted.

- The empty word $\epsilon$ is both a prefix and a suffix, i.e. $\epsilon \in S \cup R$ and $\epsilon \in E$;

- $f:(S \cup R) \cdot E \rightarrow\{+,-\}$ is a mapping function such that for every timed word (prefix) $\omega \in S \cup R$ and every timed word (suffix) $e \in E, f(\omega \cdot e)=+$ if the timed word $\omega \cdot e \in \mathcal{L}$, i.e. $\mathrm{MQ}(\omega e)=+$ and $f(\omega \cdot e)=-$ otherwise, i.e. $\mathrm{MQ}(\omega e)=-$.

Given a table $\mathcal{T}$, we define a function val $: S \cup R \rightarrow(E \rightarrow\{+,-\})$ mapping every prefix $\omega \in S \cup R$ to a value vector indexed by the suffix $e \in E$, in which each value is defined by $f(\omega \cdot e)$. Intuitively, the vectors denote different locations in a hypothesis automaton, and the suffixes are used to distinguish the locations. Here we define a function $r o w: S \cup R \rightarrow 2^{E}$ by $\operatorname{row}(\omega)=\{e \in E$ | $f(\omega \cdot e)=+\}$ for each $\omega \in S \cup R$. Hence, considering the definitions of residual real-time languages and RRTAs in Section 4, we find that row $(\omega)$ represents a subset of the residual real-time language $\omega^{-1} \mathcal{L}$ indicated by the prefix $\omega \in S \cup R$.

Therefore, following [8], in order to indicate the prime and composed residual real-time languages of $\mathcal{L}$ using the prefixes in $S \cup R$, we define prime and composed prefixes as follows.

Definition 5.2 (Prime and Composed Prefixes). Given a table $\mathcal{T}=(\Sigma, \Xi, S, R, E, f)$, a prefix $\omega \in$ $S \cup R$ is prime if

$$
\bigcup\left\{\operatorname{row}\left(\omega^{\prime}\right) \mid \operatorname{row}\left(\omega^{\prime}\right) \subsetneq \operatorname{row}(\omega)\right\} \subsetneq \operatorname{row}(\omega)
$$

with $\omega^{\prime} \in S \cup R$, otherwise composed.

In other words, a prefix $\omega \in S \cup R$ is composed if there exist $\omega_{1}, \omega_{2}, \ldots, \omega_{n} \in(S \cup R) \backslash\{\omega\}$ such that $\operatorname{row}(\omega)=\operatorname{row}\left(\omega_{1}\right) \cup \operatorname{row}\left(\omega_{2}\right) \cup \cdots \cup \operatorname{row}\left(\omega_{n}\right)$. Otherwise, the prefix $\omega$ is prime. It's worth noting that given a prime prefix $\omega \in S \cup R$ there may exist prime prefixes $s \in S$ such that row $(s) \subsetneq$ row $(\omega)$.

Example 5.3. Figure 2 shows another NRTA $\mathcal{A}$ and an instance of the real-time observation table $\mathcal{T}$ during the learning process for $\mathcal{A}$. We use the example to illustrate the definitions about real-time observation tables. The prefix set $S$ is $\{\epsilon,(a, 5.1),(a, 0),(a, 5.1)(a, 8.1)\}$, the extended prefix set $R$ is $\{(b, 0),(a, 5.1)(a, 0),(a, 5.1)(b, 0),(a, 7), \ldots\}$ and the suffix set $E$ is $\{\epsilon,(a, 8.1),(a, 8.1)(a, 8.1),(a, 15)\}$. Given a timed word $\omega \cdot e=(a, 5.1) \cdot(a, 8.1)$, we have $f(\omega \cdot e)=$ + , since it is accepted by $\mathcal{A}$. For the functions val and row, we have $\operatorname{val}((a, 5.1))=\{-,+,-,-\}$ and row $=\{(a, 8.1)\}$. The prefix $(a, 7)$ is a composed prefix since $\operatorname{row}((a, 7))=\{\epsilon,(a, 8.1)\}=$ $\operatorname{row}((a, 5.1)(a, 8.1)) \cup \operatorname{row}((a, 5.1))$. As an illustration of the note in the previous paragraph, although $(a, 5.1)$ is a prime prefix, there exists a prime prefix $(a, 0) \in S$ such that $\operatorname{row}((a, 0)) \subsetneq$ $\operatorname{row}((a, 5.1))$.

The basic idea of the learning algorithm is to find all prime prefixes. Based on Definition 4.3, there exists a set of timed words to distinguish prime residual real-time languages from each other. Thus we try to find the suffix set $E$ containing distinguishing words. Before constructing a hypothesis from a table, the learner has to ensure that the table satisfies the following readiness conditions: 
- Reduced: $\forall s \in S: s$ is prime, and $\forall s, s^{\prime} \in S: s \neq s^{\prime} \Rightarrow \operatorname{val}(s) \neq \operatorname{val}\left(s^{\prime}\right)$.

- Closed: $\forall r \in R: \operatorname{row}(r)=\bigcup\{\operatorname{row}(s) \mid s \in S \wedge \operatorname{row}(s) \subseteq \operatorname{row}(r)\}$.

- Consistent: $\forall \omega, \omega^{\prime} \in S \cup R: \operatorname{row}(\omega) \subseteq \operatorname{row}\left(\omega^{\prime}\right) \Rightarrow \operatorname{row}(\omega \cdot \boldsymbol{\sigma}) \subseteq \operatorname{row}\left(\omega^{\prime} \cdot \boldsymbol{\sigma}\right)$ if $\omega \cdot \boldsymbol{\sigma}, \omega^{\prime} \cdot \boldsymbol{\sigma} \in$ $S \cup R$, where $\sigma \in \Xi=\Sigma \times \mathbb{R}_{\geq 0}$.

- Evidence-closed: $\forall s \in S, e \in E: s \cdot e \in S \cup R$.

- Distinctness: $\forall \omega \in S \cup R, \sigma \in \Xi=\Sigma \times \mathbb{R}_{\geq 0}: \omega \cdot \sigma \in S \cup R \Rightarrow s_{i} \cdot \sigma \in S \cup R$, where $s_{i} \in\{s \in S \mid \operatorname{row}(s) \subseteq \operatorname{row}(\omega)\}$.

Since $S$ is the set of current prime prefixes, a reduced table helps to set a bijection between $S$ and prime residuals, which corresponds to Definition 4.5. A closed table ensures that for every current composed residuals indicated by $r \in R$ is composed by an union of prime residuals indicated by current prime prefixes, which corresponds to Definition 4.3 and 5.2. Given a table, if $\operatorname{row}(\omega) \subseteq \operatorname{row}\left(\omega^{\prime}\right)$, it shows a relation that the residual language indicated by $\omega$ is a subset of the residual language indicated by $\omega^{\prime}$. Hence if the table is consistent, after performing the same timed action $\sigma$, the relation is still maintained for the timed words $\omega \cdot \sigma$ and $\omega^{\prime} \cdot \sigma$. The above two readiness conditions are direct extensions of those in [8]. The evidence-closed condition is added and serves the same function as in $[4,5,16]$. The last condition, distinctness, is unique for this paper, and is needed to deal with nondeterministic behavior caused by timing information Given a prefix $\omega$, it represents a virtual location which combines the locations indicated by prime prefixes $s_{i} \in\{s \in S \mid \operatorname{row}(s) \subseteq \operatorname{row}(\omega)\}$. If a table contains the prefixes $s_{i} \cdot \boldsymbol{\sigma}$, it will make the residual and location indicated by $s_{i}$ distinct. Such condition prevents the hypothesis receiving a repeating counterexample and therefore ensures the termination of our learning algorithm. A realtime observation table is prepared if it satisfies the above conditions. To achieve it, we introduce the following operations.

Making $\mathcal{T}$ reduced. If $\mathcal{T}$ is not reduced, there are two cases. If there is an $s \in S$ which is not prime, there exists a set $\left\{s_{1}, s_{2}, \ldots, s_{n}\right\} \in S \backslash\{s\}$ such that $\operatorname{row}(s)=\operatorname{row}\left(s_{1}\right) \cup \operatorname{row}\left(s_{2}\right) \cup \cdots \cup \operatorname{row}\left(s_{n}\right)$. In this case we fix the table by moving $s$ to $R$. The second part of the condition, that there are no two rows in $S$ which are the same, is guaranteed by the other table operations and the process of handling counterexamples in Section 5.3.

Making $\mathcal{T}$ closed. If $\mathcal{T}$ is not closed, we find a row $r \in R$ such that $\bigcup\{\operatorname{row}(s) \mid \operatorname{row}(s) \subseteq \operatorname{row}(r)\} \neq$ $\operatorname{row}(r)$, where $s \in S$, then move $r$ from $R$ to $S$. It means that we find a new location. Additionally, for each action $\sigma \in \Sigma$, we add a new row $r \cdot(\sigma, 0)$ into $R$ and fill the table by performing membership queries $\mathrm{MQ}(r \cdot(\sigma, 0) \cdot e)$ for every suffix $e \in E$. Such an operation is important since it guarantees that at every location all actions in $\Sigma$ are enabled. Comparing with the $L^{*}$ and $N L^{*}$ algorithms, we do not add timed words $r \cdot(\sigma, \tau)$ for all $\tau \in \mathbb{R}_{\geq 0}$ to the table since the number of such timed words is infinite. The partition function in Section 5.2 will handle all clock valuations in $\mathbb{R}_{\geq 0}$.

Making $\mathcal{T}$ consistent. If $\mathcal{T}$ is not consistent, it implies that there exist at least two rows $\omega, \omega^{\prime} \in$ $S \cup R$, such that $\omega \cdot \sigma, \omega^{\prime} \cdot \sigma \in S \cup R$ for some $\sigma \in \Sigma \times \mathbb{R}_{\geq 0}$, with $\operatorname{row}(\omega) \subseteq \operatorname{row}\left(\omega^{\prime}\right)$, but $\operatorname{row}(\omega \cdot \boldsymbol{\sigma}) \nsubseteq \operatorname{row}\left(\omega^{\prime} \cdot \boldsymbol{\sigma}\right)$. Then we find a suffix $e \in E$ such that $f(\omega \cdot \boldsymbol{\sigma} \cdot e)=+$ and $f\left(\omega^{\prime} \cdot \boldsymbol{\sigma} \cdot e\right)=-$ The suffix $e$ can also be found using $\operatorname{row}(\omega) \backslash\left(\operatorname{row}(\omega) \cap \operatorname{row}\left(\omega^{\prime}\right)\right)$. One inconsistency can be fixed by adding a new suffix $\sigma \cdot e$ to $E$. Afterwards, the table is filled via membership queries.

Making $\mathcal{T}$ evidence-closed. If $\mathcal{T}$ is not evidence-closed, then we can find $s \in S$ and $e \in E$ with $s \cdot e \notin S \cup R$ and put all prefixes of $s \cdot e$ in $R$ except for those already in $S \cup R$. Similarly, we need to fill the table through membership queries.

Making $\mathcal{T}$ distinct. If $\mathcal{T}$ is not distinct, then we can find a row $\omega \in S \cup R$ with $\omega \cdot \sigma \in S \cup R$ for some $\sigma \in \sum \times \mathbb{R}_{\geq 0}$ but not all $s_{i} \cdot \sigma$ in $S \cup R$, where $s_{i} \in\{s \in S \mid \operatorname{row}(s) \subseteq \operatorname{row}(\omega)\}$. Then the table 


\begin{tabular}{r|cc}
\hline $\mathcal{T}_{2}$ & $\epsilon$ & $(a, 8.1)$ \\
\hline$\epsilon$ & - & - \\
\hline$(a, 0)$ & - & - \\
$(b, 0)$ & - & - \\
$(a, 5.1)$ & - & + \\
$(a, 5.1)(a, 8.1)$ & + & - \\
\hline
\end{tabular}

\begin{tabular}{r|lc}
\hline $\mathcal{T}_{3}$ & $\epsilon$ & $(a, 8.1)$ \\
\hline$\epsilon$ & - & - \\
$(a, 5.1)$ & - & + \\
\hline$(a, 0)$ & - & - \\
$(b, 0)$ & - & - \\
$(a, 5.1)(a, 8.1)$ & + & - \\
$(a, 5.1)(a, 0)$ & - & - \\
$(a, 5.1)(b, 0)$ & - & - \\
\hline
\end{tabular}

\begin{tabular}{r|lc}
\hline $\mathcal{T}_{4}$ & $\epsilon$ & $(a, 8.1)$ \\
\hline$\epsilon$ & - & - \\
$(a, 5.1)$ & - & + \\
\hline$(a, 0)$ & - & - \\
$(b, 0)$ & - & - \\
$(a, 5.1)(a, 8.1)$ & + & - \\
$(a, 5.1)(a, 0)$ & - & - \\
$(a, 5.1)(b, 0)$ & - & - \\
$(a, 8.1)$ & - & + \\
\hline
\end{tabular}

\begin{tabular}{r|ccc}
\hline $\mathcal{T}_{5}$ & $\epsilon$ & $(a, 8.1)$ & $(a, 8.1)(a, 8.1)$ \\
\hline$\epsilon$ & - & - & + \\
$(a, 5.1)$ & - & + & - \\
\hline$(a, 0)$ & - & - & - \\
$(b, 0)$ & - & - & - \\
$(a, 5.1)(a, 8.1)$ & + & - & - \\
$(a, 5.1)(a, 0)$ & - & - & - \\
$(a, 5.1)(b, 0)$ & - & - & - \\
$(a, 8.1)$ & - & + & - \\
\hline
\end{tabular}

Fig. 3. The table instances used in Example 5.4 to illustrate the readiness conditions and the corresponding operations. These table instances are generated during the learning process of the running example presented in Section 5.6.

can be fixed by adding all such $s_{i} \cdot \sigma$ to $R$. Similarly, the table needs to be filled via membership queries. Note that we need to perform this operation no matter if $\omega$ is prime or composed.

A table may need several rounds of the above operations before being prepared (cf. Algorithm 1). The following example illustrates the readiness conditions and the corresponding operations.

Example 5.4. As shown in Figure 3, there are four table instances $\mathcal{T}_{2}, \mathcal{T}_{3}, \mathcal{T}_{4}$ and $\mathcal{T}_{5}$.

$\mathcal{T}_{2}$ is not closed since there exists a row $(a, 5.1) \in R$ such that $\bigcup\{\operatorname{row}(\epsilon)\}=\emptyset \neq \operatorname{row}((a, 5.1))$. In order to repair it, we move the row $(a, 5.1)$ from $R$ to $S$ and then add $(a, 5.1) \cdot(a, 0)$ and $(a, 5.1) \cdot(b, 0)$ to $R$. After making membership queries, we get $\mathcal{T}_{3}$.

$\mathcal{T}_{3}$ is not distinct. It can be shown as follows. Let $\omega \cdot \sigma=(a, 5.1) \cdot(a, 8.1)$. Then we can build the set $\{\epsilon,(a, 5.1)\}$ since $\operatorname{row}(\epsilon) \subseteq \operatorname{row}((a, 5.1))$ and $\operatorname{row}((a, 5.1)) \subseteq \operatorname{row}((a, 5.1))$. However, $\epsilon \cdot(a, 8.1)$ is not in $S \cup R$. Hence, we add $\epsilon \cdot(a, 8.1)$ into $R$ to repair it. This results in table $\mathcal{T}_{4}$. Actually, since $\mathcal{T}_{3}$ is also not evidence-closed, $\epsilon \cdot(a, 8.1)$ can also be added into $R$ in the process of making $\mathcal{T}_{3}$ evidence-closed. It depends on the checking order of the conditions.

$\mathcal{T}_{4}$ is not consistent, since $\operatorname{row}(\epsilon) \subseteq \operatorname{row}((a, 5.1))$ but $\operatorname{row}(\epsilon \cdot(a, 8.1)) \nsubseteq \operatorname{row}((a, 5.1) \cdot(a, 8.1))$. To repair it, we find the suffix $(a, 8.1) \in E$ with $f(\epsilon \cdot(a, 5.1) \cdot(a, 8.1))=+$ and $f((a, 5.1) \cdot(a, 8.1)$. $(a, 5.1))=-$, then add $(a, 8.1) \cdot(a, 8.1)$ to $E$ as a new suffix. This results in table $\mathcal{T}_{5}$. It is not hard to find that $\mathcal{T}_{5}$ is not closed and we can move $(a, 5.1)(a, 8.1)$ from $R$ to $S$ to repair it.

\subsection{Constructing a Hypothesis from a Prepared Table $\mathcal{T}$}

Once the learner obtains a prepared table $\mathcal{T}$, a hypothesis NRTA can be constructed in two steps. The learner first constructs an NFA $M$ from $\mathcal{T}$, and then transforms it to an NRTA $\mathcal{H}$ as the current hypothesis. We describe each of the two steps in turn.

Construction of NFA $M$. Given a prepared real-time observation table $\mathcal{T}=(\Sigma, \Xi, S, R, E, f)$, the learner builds an NFA $M=\left(Q_{M}, \Sigma_{M}, \Delta_{M}, Q_{M}^{0}, F_{M}\right)$ as follows:

- the finite set of locations $Q_{M}=\left\{q_{\text {val }}(s) \mid s \in S\right\}$;

- the abstract alphabet $\Sigma_{M}=\{\sigma \in \Xi \mid \omega \cdot \sigma \in S \cup R \wedge \operatorname{row}(\omega) \in\{\operatorname{row}(s) \mid s \in S\}\}$;

- the transition relation $\Delta_{M}=\left\{\left(q_{\operatorname{val}(\omega)}, \sigma, q_{\text {val }\left(s^{\prime}\right)}\right) \mid \omega \cdot \sigma \in S \cup R \wedge \operatorname{row}(\omega) \in\{\operatorname{row}(s) \mid s \in\right.$ $\left.S\} \wedge \operatorname{row}\left(s^{\prime}\right) \subseteq \operatorname{row}(\omega \cdot \sigma) \wedge s^{\prime} \in S\right\}$; (The corresponding transition function is denoted as $\hat{\delta}$ );

- the set of initial locations $Q_{M}^{0}=\left\{q_{\text {val }(s)} \mid \operatorname{row}(s) \subseteq \operatorname{row}(\epsilon)\right.$ for $\left.s \in S \wedge \epsilon \in S \cup R\right\}$;

- the set of accepting locations $F_{M}=\left\{q_{\text {val }(s)} \mid f(s \cdot \epsilon)=+\right.$ for $\left.s \in S \wedge \epsilon \in E\right\}$.

If $\mathcal{T}$ is a prepared table, the NFA $M$ is well-defined. For the set of locations, we let each current prime prefix in $S$ to represent a location. The transitions are built as follows. For each timed word $\omega \cdot \sigma \in S \cup R$, since $\mathcal{T}$ is prefix-closed, we know $\omega$ is also in $S \cup R$. If $\omega$ is prime, then $\operatorname{row}(\omega) \in$ $\{\operatorname{row}(s) \mid s \in S\}$, and we add a transition $\left(q_{\operatorname{val}(\omega)}, \sigma, q_{\operatorname{val}\left(s^{\prime}\right)}\right)$ for each $s^{\prime} \in S$ with $\operatorname{row}\left(s^{\prime}\right) \subseteq$ $\operatorname{row}(\omega \cdot \sigma)$. If $\omega$ is composed, we have $\operatorname{row}(\omega) \notin\{\operatorname{row}(s) \mid s \in S\}$, so no transition comes directly 
from $\omega$. However, since $\mathcal{T}$ is distinct, if row $(\omega)$ decomposes as $\operatorname{row}\left(s_{1}\right) \cup \operatorname{row}\left(s_{2}\right) \cup \cdots \cup \operatorname{row}\left(s_{n}\right)$, where each $s_{i} \in S$, then we have added all $s_{i} \cdot \sigma$ for $1 \leq i \leq n$ into $R$, and it suffices to build transitions using row $\left(s_{i}\right)$. The set $\Sigma_{M}$ collects all timed actions $\sigma$ which can trigger transitions, viewing a timed action $\sigma$ as an abstract action in NFA $M$. For initial locations, the formula $Q_{M}^{0}=$ $\left\{q_{\operatorname{val}(s)} \mid \operatorname{row}(s) \subseteq \operatorname{row}(\epsilon)\right.$ for $\left.s \in S \wedge \epsilon \in S \cup R\right\}$ means that the virtual initial location (reached by $\epsilon$ ) is composed from several actual locations. Hence, the prefix $\epsilon$ is not in $S$ if and only if it is a composed prefix. The set of accepting locations collects all locations indicated by the prime prefixes which are in $\mathcal{L}$.

Definition 5.5 (Compatibility). Let $\mathcal{T}=(\Sigma, \Xi, S, R, E, f)$ be a prepared table, and $M=$ $\left(Q_{M}, \Sigma_{M}, \Delta_{M}, Q_{M}^{0}, F_{M}\right)$ be an NFA. We say $M$ is compatible with $\mathcal{T}$ if for every timed word $\omega \cdot e \in(S \cup R) \cdot E, M$ accepts $\omega \cdot e$ if and only if $f(\omega \cdot e)=+$.

According to the learning algorithms $[4,5,16]$ for deterministic automata, we need to prove that NFA $M$ is compatible with table $\mathcal{T}$. However, as pointed out in [8], this property does not necessarily hold for the constructed NFA in every round. Instead, it is shown in [8] that $M$ satisfies several weaker properties, which is still sufficient for proving correctness of the algorithm. Further it is shown that if $M$ is compatible with $\mathcal{T}$, then $M$ is a canonical RFSA. Compared to [8], we defined several extra readiness conditions and modified the process for handling counterexamples, so the statement and proofs of the lemmas are slightly modified. The lemmas are as follows.

Lemma 5.6 ([8, Lemma 2]). Let $\mathcal{T}=(\Sigma, \Xi, S, R, E, f)$ be a prepared table and $M=\left(Q_{M}, \Sigma_{M}\right.$, $\left.\Delta_{M}, Q_{M}^{0}, F_{M}\right)$ be the constructed NFA. For every timed word $s \cdot e \in S \cdot E, f(s \cdot e)=+$ iffe $e \in L_{q_{v a l(s)}}$, where $L_{q_{\text {val (s) }}}$ is the language of location $q_{\text {val }(s)}$, i.e. $\hat{\delta}\left(q_{\text {val }(s)}, e\right) \cap F_{M} \neq \emptyset$. Moreover, $f(\epsilon \cdot e)=+$ iff $e \in L(M)$, where $L(M)$ is the recognized language of NFA $M$.

Proof. Suppose $e=\epsilon$, we have $f(s \cdot e)=f(s \cdot \epsilon)=+$ iff $q_{v a l(s)} \in F_{M}$ by the definition of $F_{M}$. Hence, we have $f(s \cdot \epsilon)=+$ iff $\epsilon \in L_{q_{v a l(s)}}$.

Now suppose $e=\sigma \cdot e^{\prime}$. Since $\mathcal{T}$ is evidence-closed, we have $s \cdot \sigma \in S \cup R$. Since $E$ is suffixclosed, we have $e^{\prime} \in E$. By the definition of $\Delta_{M}$, there exist transitions with source location $q_{v a l}(s)$ and action $\sigma$, so that $\sigma \in \Sigma_{M}$. We prove the lemma by induction on the length of $e \in E$.

If $f(s \cdot e)=+$, then $f\left((s \cdot \boldsymbol{\sigma}) \cdot e^{\prime}\right)=f\left(s \cdot\left(\sigma \cdot e^{\prime}\right)\right)=+$. We wish to find $s^{\prime} \in S$ such that $f\left(s^{\prime} \cdot e^{\prime}\right)=+$. If $s \cdot \sigma \in S$, it suffices to set $s^{\prime}=s \cdot \boldsymbol{\sigma}$. Otherwise, we have $s \cdot \sigma \in R$. Since $f\left((s \cdot \boldsymbol{\sigma}) \cdot e^{\prime}\right)=+$, there exists at least one $s^{\prime} \in S$ such that $f\left(s^{\prime} \cdot e^{\prime}\right)=+$ and $\operatorname{row}\left(s^{\prime}\right) \subseteq \operatorname{row}(s \cdot \sigma)$, and it suffices to take that $s^{\prime}$. By induction hypothesis, $e^{\prime} \in L_{q_{v a l\left(s^{\prime}\right)}}$. By the definition of $\Delta_{M}$, there is a transition from $q_{v a l(s)}$ to $q_{\text {val (s') }}$ with action $\sigma$. Hence, the suffix $e=\sigma \cdot e^{\prime}$ is in $L_{q_{v a l(s)}}$. In particular, if $\epsilon \in S$ and $f(\epsilon \cdot e)=+$, we have shown that $e \in L_{q_{v a l}(\epsilon)}$. By the definition of $Q_{M}^{0}$, if $\epsilon \in S, L_{q_{v a l}(\epsilon)} \subseteq L(M)$ then $e \in L(M)$. If $\epsilon \in R$, by the definition of $Q_{M}^{0}$, every s such that $q_{v a l(s)} \in Q_{M}^{0}$ satisfies row $(s) \subseteq$ row $(\epsilon)$. Additionally, since $\mathcal{T}$ is closed, then we can find at least one $s \in S$ such that $q_{\text {val }(s)} \in Q_{M}^{0}$ and $f(s \cdot e)=+$. It follows $e \in L(M)$.

For the inverse direction, suppose $e=\sigma \cdot e^{\prime}$ and $f(s \cdot e)=-$. We have $f\left((s \cdot \sigma) \cdot e^{\prime}\right)=f\left(s \cdot\left(\sigma \cdot e^{\prime}\right)\right)=$ - . We wish to show $f\left(s^{\prime} \cdot e^{\prime}\right)=-$ for every $s^{\prime} \in S$ satisfying $\operatorname{row}\left(s^{\prime}\right) \subseteq \operatorname{row}(s \cdot \sigma)$. Since $\mathcal{T}$ is closed, we have $\operatorname{row}(s \cdot \sigma)=\bigcup\left\{\operatorname{row}\left(s^{\prime}\right) \mid s^{\prime} \in S \wedge \operatorname{row}\left(s^{\prime}\right) \subseteq \operatorname{row}(s \cdot \sigma)\right\}$. Since $f\left((s \cdot \sigma) \cdot e^{\prime}\right)=-$ and $\operatorname{row}\left(s^{\prime}\right) \subseteq \operatorname{row}(s \cdot \sigma)$, then $f\left(s^{\prime} \cdot e^{\prime}\right)=-$ for every such $s^{\prime}$. By the induction hypothesis, $e^{\prime} \notin L_{q_{v a l}\left(s^{\prime}\right)}$ for all $s^{\prime} \in S$ satisfying $\operatorname{row}\left(s^{\prime}\right) \subseteq \operatorname{row}(s \cdot \sigma)$. By the definition of $\Delta_{M}$, there exists no transition from $q_{\text {val }(s)}$ to $q_{\text {val }\left(s^{\prime}\right)}$ with action $\sigma$. Hence, the suffix $e=\sigma \cdot e^{\prime}$ is not in $L_{q_{v a l(s)}}$. Moreover, we can prove if $f(\epsilon \cdot e)=-$ then $e \notin L(M)$.

Combining the two directions, we have $f(s \cdot e)=+\operatorname{iff} e \in L_{q_{v a l(s)}}$ and $f(\epsilon \cdot e)=+\operatorname{iff} e \in L(M)$. 
LeMmA 5.7 ([8, LEMMA 3]). Let $\mathcal{T}=(\Sigma, \Xi, S, R, E, f)$ be a prepared table and $M=\left(Q_{M}, \Sigma_{M}, \Delta_{M}\right.$, $\left.Q_{M}^{0}, F_{M}\right)$ be the constructed $N F A$. For $s, s^{\prime} \in S$, row $(s) \subseteq \operatorname{row}\left(s^{\prime}\right)$ if and only if $L_{q_{\text {val }(s)}} \subseteq L_{q_{\text {val }}\left(^{\prime}\right)}$.

Proof. If $\operatorname{row}(s) \subseteq \operatorname{row}\left(s^{\prime}\right)$ and suppose $\omega \in L_{q_{v a l(s)}}$, we need to prove $\omega \in L_{\left.q_{\text {val }} s^{\prime}\right)}$. If $\omega=\epsilon$, we have $f(s \cdot \epsilon)=+$. Since $\operatorname{row}(s) \subseteq \operatorname{row}\left(s^{\prime}\right), f\left(s^{\prime} \cdot \epsilon\right)=+$. By the definition of $F_{M}, q_{\text {val }\left(s^{\prime}\right)} \in F_{M}$. Hence, $\omega=\epsilon \in L_{q_{\text {val }\left(s^{\prime}\right)}}$. If $\omega=\sigma \cdot \omega^{\prime}$, where $\sigma \in \Sigma_{M}$ and $\omega \in L_{q_{v a l(s)}}$, then $\hat{\delta}\left(q_{\text {val }(s)}, \sigma \cdot \omega^{\prime}\right) \cap F_{M} \neq \emptyset$. Hence there is $s^{\prime \prime} \in S$ with $q_{\text {val }\left(s^{\prime \prime}\right)} \in \hat{\delta}\left(q_{\text {val }(s)}, \sigma\right)$ and $\hat{\delta}\left(q_{\text {val }\left(s^{\prime \prime}\right)}, \omega^{\prime}\right) \cap F_{M} \neq \emptyset$. Based on the definition of $\Delta_{M}, \operatorname{row}\left(s^{\prime \prime}\right) \subseteq \operatorname{row}(s \cdot \sigma)$. Since $\mathcal{T}$ is consistent, we also have $\operatorname{row}(s \cdot \sigma) \subseteq \operatorname{row}\left(s^{\prime} \cdot \sigma\right)$. Therefore, $\operatorname{row}\left(s^{\prime \prime}\right) \subseteq \operatorname{row}\left(s^{\prime} \cdot \sigma\right)$. Thus, $s^{\prime \prime} \in \hat{\delta}\left(q_{\operatorname{val}\left(s^{\prime}\right)}, \sigma\right)$, which implies $\omega=\sigma \cdot \omega^{\prime} \in L_{q_{\text {val }}\left(^{\prime}\right)}$.

For the inverse direction, suppose $\operatorname{row}(s) \nsubseteq \operatorname{row}\left(s^{\prime}\right)$, then there exists $e \in E$ such that $f(s \cdot e)=+$ while $f\left(s^{\prime} \cdot e\right)=-$. By Lemma 5.6, $e \in L_{q_{\text {val(s) }}}$ and $e \notin L_{q_{\text {val }\left(s^{\prime}\right)}}$. Therefore, $L_{q_{\text {val }(s)}} \nsubseteq L_{q_{\text {val }\left(s^{\prime}\right)}}$.

Lemma 5.8 ([8, Lemma 4]). Let $\mathcal{T}=(\Sigma, \Xi, S, R, E, f)$ be a prepared table and suppose the constructed NFA $M=\left(Q_{M}, \Sigma_{M}, \Delta_{M}, Q_{M}^{0}, F_{M}\right)$ is compatible with $\mathcal{T}$. For every $\omega \in S \cup R$, if $\omega$ is prime, then $q_{\operatorname{val}(\omega)} \in \hat{\delta}\left(Q_{M}^{0}, \omega\right)$.

Proof. Suppose $q_{\text {val }(\omega)} \notin \hat{\delta}\left(Q_{M}^{0}, \omega\right)$. Since $\omega$ is prime, then there is a prefix $s \in S$ with $\operatorname{row}(s)=$ row $(\omega)$. Thus, $q_{v a l(s)} \notin \hat{\delta}\left(Q_{M}^{0}, s\right)$. For all $s^{\prime} \in S$ satisfying $q_{v a l\left(s^{\prime}\right)} \in \hat{\delta}\left(Q_{M}^{0}, \omega\right)=\hat{\delta}\left(Q_{M}^{0}, s\right)$, we have $\operatorname{row}\left(s^{\prime}\right) \subseteq \operatorname{row}(s)$. By Lemma 5.7, $L_{q_{v a l\left(s^{\prime}\right)}} \subseteq L_{q_{v a l(s)}}$. As $q_{v a l(s)} \notin \hat{\delta}\left(Q_{M}^{0}, s\right)$, there exists $e \in E$ such that $f(s \cdot e)=+$ and for all $q_{\text {val }\left(s^{\prime}\right)} \in \hat{\delta}\left(Q_{M}^{0}, s\right), f\left(s^{\prime} \cdot e\right)=-$ which implies $e \notin L_{q_{v a l\left(s^{\prime}\right)}}$. Thus, $e \notin L_{q_{v a l(s)}}$ and then $s \cdot e \notin L(M)$ while $f(s \cdot e)=+$. This contradicts the assumption that $M$ is compatible with $\mathcal{T}$. Hence, we have $q_{\text {val }(\omega)} \in \hat{\delta}\left(Q_{M}^{0}, \omega\right)$.

After constructing an NFA $M$ from a prepared table, the learner can transform it to a hypothesis NRTA $\mathcal{H}=\left(Q, \Sigma, \Delta, Q_{0}, F\right)$ by using the following partition function.

Definition 5.9 (Partition Function [4]). Given a list of clock valuations $\ell=\tau_{0}, \tau_{1}, \ldots, \tau_{n}$ with $0=\tau_{0}<\tau_{1} \cdots<\tau_{n}$, and $\left\lfloor\tau_{i}\right\rfloor \neq\left\lfloor\tau_{j}\right\rfloor$ if $\tau_{i}, \tau_{j} \in \mathbb{R}_{\geq 0} \backslash \mathbb{N}$ and $i \neq j$ for all $1 \leq i, j \leq n$, let $\tau_{n+1}=\infty$, then a partition function $P(\cdot)$ mapping $\ell$ to a set of intervals $\left\{I_{0}, I_{1}, \ldots, I_{n}\right\}$, which is a partition of $\mathbb{R}_{\geq 0}$, is defined as

$$
I_{i}= \begin{cases}{\left[\tau_{i}, \tau_{i+1}\right),} & \text { if } \tau_{i} \in \mathbb{N} \wedge \tau_{i+1} \in \mathbb{N} ; \\ \left(\left\lfloor\tau_{i}\right\rfloor, \tau_{i+1}\right), & \text { if } \tau_{i} \in \mathbb{R}_{\geq 0} \backslash \mathbb{N} \wedge \tau_{i+1} \in \mathbb{N} ; \\ {\left[\tau_{i},\left\lfloor\tau_{i+1}\right\rfloor\right],} & \text { if } \tau_{i} \in \mathbb{N} \wedge \tau_{i+1} \in \mathbb{R}_{\geq 0} \backslash \mathbb{N} ; \\ \left(\left\lfloor\tau_{i}\right\rfloor,\left\lfloor\tau_{i+1}\right\rfloor\right], & \text { if } \tau_{i} \in \mathbb{R}_{\geq 0} \backslash \mathbb{N} \wedge \tau_{i+1} \in \mathbb{R}_{\geq 0} \backslash \mathbb{N} .\end{cases}
$$

Construction of hypothesis $\mathcal{H}$. The hypothesis NRTA $\mathcal{H}=\left(Q, \Sigma, \Delta, Q_{0}, F\right)$ can be constructed by the following steps. First, let $Q=Q_{M}, Q_{0}=Q_{M}^{0}, F=F_{M}$ and let $\Sigma$ be the given alphabet as in $\mathcal{T}$. For every location $q \in Q_{M}$ and every action $\sigma \in \Sigma$, we find a set of clock valuations $\Psi_{q, \sigma}=\left\{\tau \mid\left(q,(\sigma, \tau), q^{\prime}\right) \in \Delta_{M}\right\}$. Then we apply the partition function $P$ to the result of sorting the elements in $\Psi_{q, \sigma}$ to get $k$ intervals, written as $I_{1}, \ldots, I_{k}$, where $k=\left|\Psi_{q, \sigma}\right|$. These intervals satisfy $\tau_{i} \in I_{i}$ for any $1 \leq i \leq k$. Consequently, for every $\left(q,\left(\sigma, \tau_{i}\right), q^{\prime}\right) \in \Delta_{M}$, a fresh transition $\left(q, \sigma, I_{i}, q^{\prime}\right)$ is added to $\Delta$. The construction build a bijection between $\Delta_{M}$ and $\Delta$.

Since NFA $M$ is not always compatible with $\mathcal{T}$, the constructed NRTA $\mathcal{H}$ is not always compatible with $\mathcal{T}$ either, i.e., it does not hold that for all $\omega \cdot e \in(S \cup R) \cdot E, \mathcal{H}$ accepts $\omega \cdot e$ if and only if $f(\omega \cdot e)=+$.

However, the following lemma shows that the two automata $\mathcal{H}$ and $M$ are compatible with each other w.r.t. all $\omega \in S \cup R$ which can be generated by $M$. 

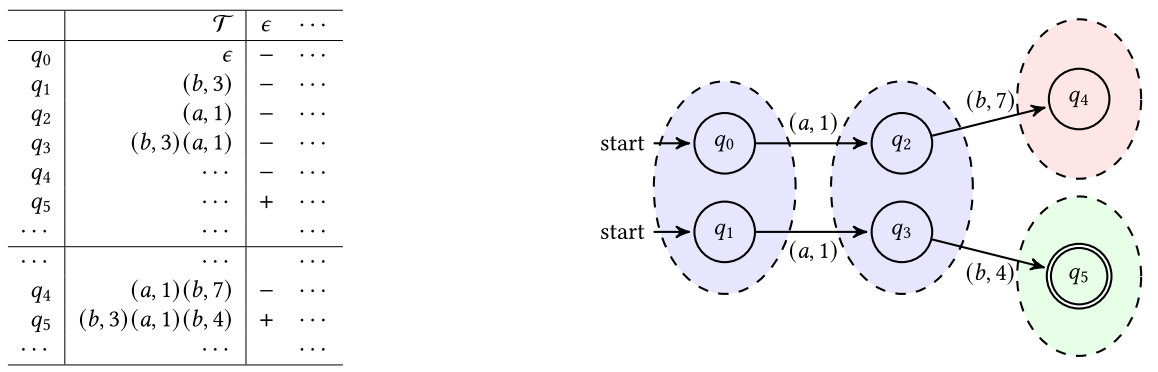

Fig. 4. An example without the distinctness condition.

LEMmA 5.10. Given a NFA $M=\left(Q_{M}, \Sigma_{M}, \Delta_{M}, Q_{M}^{0}, F_{M}\right)$ generated from a prepared table $\mathcal{T}$, and the hypothesis NRTA $\mathcal{H}=\left(Q, \Sigma, \Delta, Q_{0}, F\right)$ transformed from $M$. For all $\omega \in S \cup R$ which can be generated by $M$, we have $\mathcal{H}$ and $M$ share the same set of runs by reading $\omega$.

Proof. Suppose $\omega=\epsilon$, then $\mathcal{H}$ and $M$ share the same set of runs since $Q_{0}=Q_{M}^{0}$. Suppose $\omega=\omega^{\prime} \cdot\left(\sigma_{k}, \tau_{k}\right)$ is a timed word which can be generated by $M$. Since $\omega \in S \cup R$, then $\omega^{\prime} \in S \cup R$. By induction, we have $M$ and $\mathcal{H}$ share the same set of runs by reading $\omega^{\prime}$. Let $\left\{q_{1}, q_{2}, \ldots, q_{m}\right\}=$ $\hat{\delta}\left(Q_{M}^{0}, \omega^{\prime}\right)$ in $M$, then $\left\{q_{1}, q_{2}, \ldots, q_{m}\right\}=\delta\left(Q_{0}, \omega^{\prime}\right)$ in $\mathcal{H}$ by the inductive hypothesis. Let Source be a subset of $\left\{q_{1}, q_{2}, \ldots, q_{m}\right\}$ such that for each $q \in$ Source there exists $s \in S$ with $q=q_{\text {val }}(s)$ and $s \cdot\left(\sigma_{k}, \tau_{k}\right) \in S \cup R$. For $\omega$, there are also prime rows $s \in S$ with $\operatorname{row}(s) \subseteq \operatorname{row}(\omega)$. Suppose $\left\{q_{1}^{\prime}, q_{2}^{\prime}, \ldots, q_{n}^{\prime}\right\}=\hat{\delta}\left(Q_{M}^{0}, \omega\right)$, denoted as Target. Let Seg be the set of segments going from Source to Target which can occur as the last segment of a run in $M$ by reading $\omega$. Let Seg' be its counterpart in $\mathcal{H}$. If $\mathcal{H}$ and $M$ do not share the same set of runs by reading $\omega$, there are two possibilities:

1. Suppose there is a segment $q \underset{\tau_{k}}{\stackrel{\sigma_{k}}{\longrightarrow}} q^{\prime}$ in Seg' but not in Seg. If $q \notin\left\{q_{1}, q_{2}, \ldots, q_{m}\right\}$, then $q$ can be reached by a run reading $\omega^{\prime}$ in $\mathcal{H}$ but not in $M$. This is a contradiction to the induction hypothesis. If $q^{\prime} \notin\left\{q_{1}^{\prime}, q_{2}^{\prime}, \ldots, q_{n}^{\prime}\right\}$, then $q^{\prime}$ can be reached by a run reading $\omega$ in $\mathcal{H}$ but not in $M$. This contradicts the bijection between $\Delta_{M}$ and $\Delta$ (and each transition in $\Delta$ is obtained by extending the corresponding transition in $\Delta_{M}$ to an interval).

If $q \in\left\{q_{1}, q_{2}, \ldots, q_{m}\right\}$ and $q \notin$ Source, then there exists $s \in S$ such that $q=q_{\text {val }(s)}$, but $s \cdot\left(\sigma_{k}, \tau_{k}\right) \notin S \cup R$ and $s \cdot\left(\sigma_{k}, \tau_{k}^{\prime}\right) \in S \cup R$, where $\llbracket \tau_{k}^{\prime} \rrbracket \neq \llbracket \tau_{k} \rrbracket$ and $\tau_{k}^{\prime}<\tau_{k}$. As it leads to a transition $\left(q_{i}, \sigma_{k}, I_{k}^{\prime}, q_{j}^{\prime}\right)$, where $I_{k}^{\prime}=\left[\left\lfloor\tau_{k}^{\prime}\right\rfloor, ?\right)$ or $I_{k}^{\prime}=\left(\left\lfloor\tau_{k}^{\prime}\right\rfloor, ?\right), \tau_{k}$ satisfies the guard $I_{k}^{\prime}$ if the upper-bound of $I_{k}^{\prime}$ is greater than $\tau_{k}$. However, $s \cdot\left(\sigma_{k}, \tau_{k}\right) \notin S \cup R$ contradicts the distinctness condition. Since $\omega=\omega^{\prime} \cdot\left(\sigma_{k}, \tau_{k}\right) \in S \cup R$, then $s \cdot\left(\sigma_{k}, \tau_{k}\right) \in S \cup R$ for all $\operatorname{row}(s) \subseteq \operatorname{row}\left(\omega^{\prime}\right)$. Then the upper-bound of $I_{k}^{\prime}$ will be less than $\tau_{k}$, as the new transition is $\left(q_{i}, \sigma_{k}, I_{k}^{\prime}, q_{j}^{\prime}\right)$, where $I_{k}^{\prime}=\left[\left\lfloor\tau_{k}^{\prime}\right\rfloor,\left\lfloor\tau_{k}\right\rfloor\right)$ or $I_{k}^{\prime}=\left(\left\lfloor\tau_{k}^{\prime}\right\rfloor,\left\lfloor\tau_{k}\right\rfloor\right)$.

2. Suppose there is a segment $q \underset{\tau_{k}}{\stackrel{\sigma_{k}}{\longrightarrow}} q^{\prime}$ in Seg but not in $S_{e g}$. This is again a contradiction to the bijection between $\Delta_{M}$ and $\Delta$.

Hence, by induction, $\mathcal{H}$ and $M$ have the same set of runs by reading $\omega$.

Thanks to the distinctness condition and the bijection between $\Delta_{M}$ and $\Delta$, we can guarantee the compatibility between $\mathcal{H}$ and $M$. The following example illustrates the situation without the distinctness condition.

Example 5.11. In Figure 4, the dashed ellipses represent all ending locations after reading a timed word. There are two initial locations $q_{0}$ and $q_{1}$ of $M . M$ can reach $q_{2}$ and $q_{3}$ after reading 


\begin{tabular}{r|lc}
\hline $\mathcal{T}$ & $\epsilon$ & $(a, 5)$ \\
\hline$\epsilon$ & - & - \\
$(a, 2)$ & - & + \\
$(a, 2)(a, 5)$ & + & - \\
\hline$(a, 0)$ & - & - \\
$(b, 0)$ & - & - \\
$(a, 2)(a, 0)$ & - & - \\
$(a, 2)(b, 0)$ & - & - \\
$(a, 5)$ & - & - \\
$(a, 2)(a, 5)(a, 0)$ & - & - \\
$(a, 2)(a, 5)(b, 0)$ & - & - \\
$(a, 2)(a, 5)(a, 5)$ & - & - \\
\hline
\end{tabular}
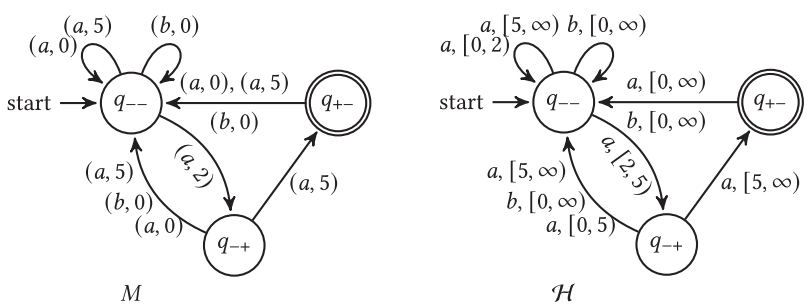

Fig. 5. A prepared table $\mathcal{T}$, the corresponding NFA $M$ and the hypothesis NRTA $\mathcal{H}$.

timed action $(a, 1) . q_{2}$ and $q_{3}$ are indicated by $(a, 1)$ and $(b, 3)(a, 1)$ respectively. $M$ reaches a nonaccepting location $q_{4}$ from $q_{2}$ after reading $(b, 7)$ and reaches an accepting location $q_{5}$ from $q_{3}$ after reading $(b, 4)$. As $\operatorname{row}((b, 3)(a, 1)) \subseteq \operatorname{row}((a, 1))$ and $(b, 3)(a, 1) \cdot(b, 7) \notin S \cup R \wedge(b, 3)(a, 1) \cdot(b, 4) \in$ $S \cup R$, where $\llbracket 4 \rrbracket \neq \llbracket 7 \rrbracket \wedge 4<7$, it follows that $\mathcal{H}$ and $M$ do not share the same set of runs for $(a, 1)(b, 7)$ by Lemma 5.10 . Thus, in the transformed $\mathcal{H}$, there exist two transitions $\left(q_{3}, b,[4, \infty), q_{5}\right)$ and $\left(q_{2}, b,[7, \infty), q_{4}\right)$. Obviously, $(a, 1)(b, 7)$ is accepted by $\mathcal{H}$ as there exists a $\rho=q_{1} \underset{1}{\stackrel{a}{\rightarrow}} q_{3} \underset{7}{\rightarrow} q_{5}$ with $q_{5} \in F$. Thus, $\mathcal{H}$ is not compatible with $M$. What's worse, if $(a, 1)(b, 7)$ is added by a negative counterexample, $(a, 1)(b, 7)$ will be a repeating counterexample since $\mathcal{H}$ is not compatible with $M$.

However, $\operatorname{row}((b, 3)(a, 1)) \subseteq \operatorname{row}((a, 1))$ since $M$ can reach $q_{3}$ by reading $(a, 1)$. With the distinctness condition, $(b, 3)(a, 1) \cdot(a, 7)$ should be added in $R$. Thus, there must exist a transition $\left(q_{3},(b, 7), q_{\text {? }}\right)$ in $M$ for every $q_{\text {? }} \in \hat{\delta}\left(q_{3},(b, 7)\right)$. What's more, $\hat{\delta}\left(q_{3},(b, 7)\right) \cap F_{M}=\emptyset$ as $(a, 1)(a, 7)$ is not accepted by $M$. After the hypothesis construction, there exist new transitions $\left(q_{3}, b,[4,7), q_{5}\right)$ (corresponding to $\left.\left(q_{3},(b, 4), q_{5}\right)\right)$ and $\left(q_{3}, b,[7,+), q_{\text {? }}\right)$ (corresponding to $\left(q_{3},(b, 7), q_{\text {? }}\right)$ ) instead of transition $\left(q_{3}, b,[4, \infty), q_{5}\right)$. Therefore, the distinctness condition can guarantee the avoidance of repeating counterexamples.

THeOREM 5.12. If the NFA $M$ is compatible with $\mathcal{T}$, then $\mathcal{H}$ is a canonical RRTA.

Proof. (sketch) Similar to [8], $M$ will be a canonical RFSA if $M$ is compatible with $\mathcal{T}$, since $M$ satisfies Lemma 5.6, 5.7 and 5.8. Due to Lemma 5.10, $M$ and $\mathcal{H}$ share the same set of runs. Based on the definition of CRRTA and the construction of $\mathcal{H}$ from $M$, we have $\mathcal{H}$ is a canonical RRTA if $M$ is a canonical RFSA. Hence, $\mathcal{H}$ is a canonical RRTA if $M$ is compatible with $\mathcal{T}$.

Example 5.13. As shown in Figure 5, we illustrate the two steps construction from $\mathcal{T}$ to $M$ and $M$ to $\mathcal{H}$. Note that we have combined some transitions and intervals. Since there are three prefixes in $S$, NFA $M$ has three locations $q_{--}, q_{-+}$and $q_{+-}$. We show an example on building transitions according to the prefixes $(a, 2)$ and $(a, 2)(a, 5)$. The source location is determined by $q_{\text {val }((a, 2))}=q_{-+}$. Taking the action $(a, 5)$, the automaton jumps to either $q_{--}$or $q_{+-}$, since $\operatorname{row}(\epsilon) \subseteq \operatorname{row}((a, 2)(a, 5))$ and $\operatorname{row}((a, 2)(a, 5)) \subseteq \operatorname{row}((a, 2)(a, 5))$. From $M$ to $\mathcal{H}$, we take an example on recovering the guards on transitions with the source location $q_{--}$and the action $a$. We first build the set $\Psi_{q_{--}, a}=\{0,2,5\}$ and then get the intervals $[0,2),[2,5)$ and $[5, \infty)$ after applying the partition function.

\subsection{Equivalence Query and Counterexample Processing}

After constructing a hypothesis NRTA $\mathcal{H}$, the learner conducts an equivalence query to the teacher, e.g. whether $\mathcal{L}(\mathcal{H})=\mathcal{L}$. We denote the equivalence query oracle as EQ. In theory, suppose that the teacher holds an RTA $\mathcal{A}$ with $\mathcal{L}(\mathcal{A})=\mathcal{L}$. The teacher can answer the query be checking whether $\mathcal{L}(\mathcal{H})=\mathcal{L}(\mathcal{A})$. The complexity of the equivalence problem of NRTA is PSPACE-complete and can be decided by first converting NRTA to DRTA and then using classic methods to decide the inclusion problem of two DRTAs. However, it is not scalable. In [9], Bonchi and Pous introduced 
an algorithm named HKC to decide the equivalence problem of NFAs based on technique of bisimulation up to congruence. We adapt it to decide the equivalence problem of NRTAs. In practice, the equivalence query is usually done by conformance testing.

If the teacher gives a negative answer for an equivalence query, the learner can also receive a timed word $\omega$ as a counterexample for $\mathcal{L}(\mathcal{H})=\mathcal{L}$, i.e. either $\omega \in \mathcal{L}(\mathcal{H}) \wedge \omega \notin \mathcal{L}$ or $\omega \notin \mathcal{L}(\mathcal{H}) \wedge$ $\omega \in \mathcal{L}$. We call $\omega$ a negative counterexample and a positive counterexample in the two cases, respectively.

Processing counterexample: Step 1. Suppose a counterexample $\omega=\left(\sigma_{1}, \tau_{1}\right)\left(\sigma_{2}, \tau_{2}\right) \cdots\left(\sigma_{n}, \tau_{n}\right)$. First, the learner apply a normalization function norm on $\omega$ and get $\omega^{\prime}=\left(\sigma_{1}, \tau_{1}^{\prime}\right)\left(\sigma_{2}, \tau_{2}^{\prime}\right) \cdots\left(\sigma_{n}, \tau_{n}^{\prime}\right)$ as a new counterexample.

Definition 5.14 (Normalization Function [4]). A normalization function norm maps a timed word $\omega=\left(\sigma_{1}, \tau_{1}\right)\left(\sigma_{2}, \tau_{2}\right) \cdots\left(\sigma_{n}, \tau_{n}\right)$ to another timed word by resetting any clock valuation to its integer part plus a constant fractional part, i.e., $\operatorname{norm}(\omega)=\left(\sigma_{1}, \tau_{1}^{\prime}\right)\left(\sigma_{2}, \tau_{2}^{\prime}\right) \cdots\left(\sigma_{n}, \tau_{n}^{\prime}\right)$, where $\tau_{i}^{\prime}=\tau_{i}$ if $\tau_{i} \in \mathbb{N}$, and otherwise $\tau_{i}^{\prime}=\left\lfloor\tau_{i}\right\rfloor+\theta$ for some fixed constant $\theta \in(0,1)$.

The following theorem guarantees that $\omega^{\prime}$ is still a correct counterexample. In this paper, we set $\theta=0.1$. Clearly our approach works for any other value in interval $(0,1)$.

Theorem 5.15 ( [4]). If a timed word $\omega=\left(\sigma_{1}, \tau_{1}\right)\left(\sigma_{2}, \tau_{2}\right) \cdots\left(\sigma_{n}, \tau_{n}\right) \in \mathcal{L}(\mathcal{H}) \ominus \mathcal{L}$, its normalization $\omega^{\prime}=\operatorname{norm}(\omega) \in \mathcal{L}(\mathcal{H}) \ominus \mathcal{L}$, where $\ominus$ denotes the symmetric difference of two sets.

Proof. (sketch) It can be proved according to the definitions of region and NRTA.

Processing counterexample: Step 2. After the normalization, add all prefixes of $\omega^{\prime}$ to $R$ and add all suffixes of $\omega^{\prime}$ to $E$, except for those already in $S \cup R$ and $E$ respectively. The learner then fills the current table $\mathcal{T}$ by membership queries.

Our counterexample process is different from $L^{*}$ 's and $N L^{*}$ 's. All prefixes of $\omega^{\prime}$ are added in $R$, as we want to query other timed actions $(\sigma, \tau)$, where $\sigma \in \Sigma$ and $\tau \neq 0$, since we only add $s \cdot(\sigma, 0)$ to $R$ when making table closed. The query results will help to modify the guards in transitions. Likewise, we want to collect suffixes which can distinguish the prime residuals indicated by the prefixes $s \in S$, so all suffixes of a counterexample are added to $E$. In [21] and [8], it is only needed to add all suffixes of a counterexample to $E$, since $s \cdot \sigma$ for every $\sigma \in \Sigma$ is added to the table when making table closed. However, we cannot obtain this as the number of timed actions $(\sigma, \tau)$ is infinite.

This procedure can be viewed as a nondeterministic version combining the counterexample process in [4] and that in [21]. The important thing is to consider all $s \in S$ with $\operatorname{row}(s) \subseteq \operatorname{row}(\epsilon)$ which indicate multiple initial locations instead of just considering $\epsilon$. Thanks to the distinctness condition, we just need to add all prefixes of $\omega^{\prime}$ to $R$ rather than all elements in $\{s \cdot u \mid s \in$ $\left.S \wedge \operatorname{row}(s) \subseteq \operatorname{row}(\epsilon) \wedge u \in \operatorname{prefixes}\left(\omega^{\prime}\right)\right\}$ to $R$ in this step, where prefixes $\left(\omega^{\prime}\right)$ is the prefix set of $\omega^{\prime}$. Since $u$ can be viewed as $\epsilon \cdot u$, if the table is distinct, then $\epsilon \cdot u \in S \cup R \Rightarrow s_{i} \cdot u \in S \cup R$, where $s_{i} \in\{s \mid \operatorname{row}(s) \subseteq \operatorname{row}(\epsilon)\}$. Removing the condition will lead to repeating counterexamples as $\epsilon$ may be composed and thus not in $S$. As a result, adding all prefixes of counterexamples may not exclude any error in the current hypothesis. Therefore, the learning process will not terminate.

Improving Step 2 by homing sequences decomposition. In [22], Rivest and Schapire introduced a decomposition on counterexamples. We adapt it to a nondeterministic version in our counterexample processing (Step 2) as an improvement. For a normalized counterexample $\omega^{\prime}$, if $V_{q_{v a l(s)} \in Q_{0}} M Q\left(s \cdot \omega^{\prime}\right) \neq M Q\left(\omega^{\prime}\right)$, then there exists a decomposition $\omega^{\prime}=u \cdot \sigma \cdot v$ into a prefix $u$, a timed action $\sigma \in \Xi$, and a suffix $v$ such that $\bigvee_{q_{v a l(s)} \in \delta\left(Q_{0}, u\right)} M Q(s \cdot \sigma v) \neq \bigvee_{q_{v a l\left(s^{\prime}\right)} \in \delta\left(Q_{0}, u \sigma\right)} M Q\left(s^{\prime} \cdot v\right)$. 


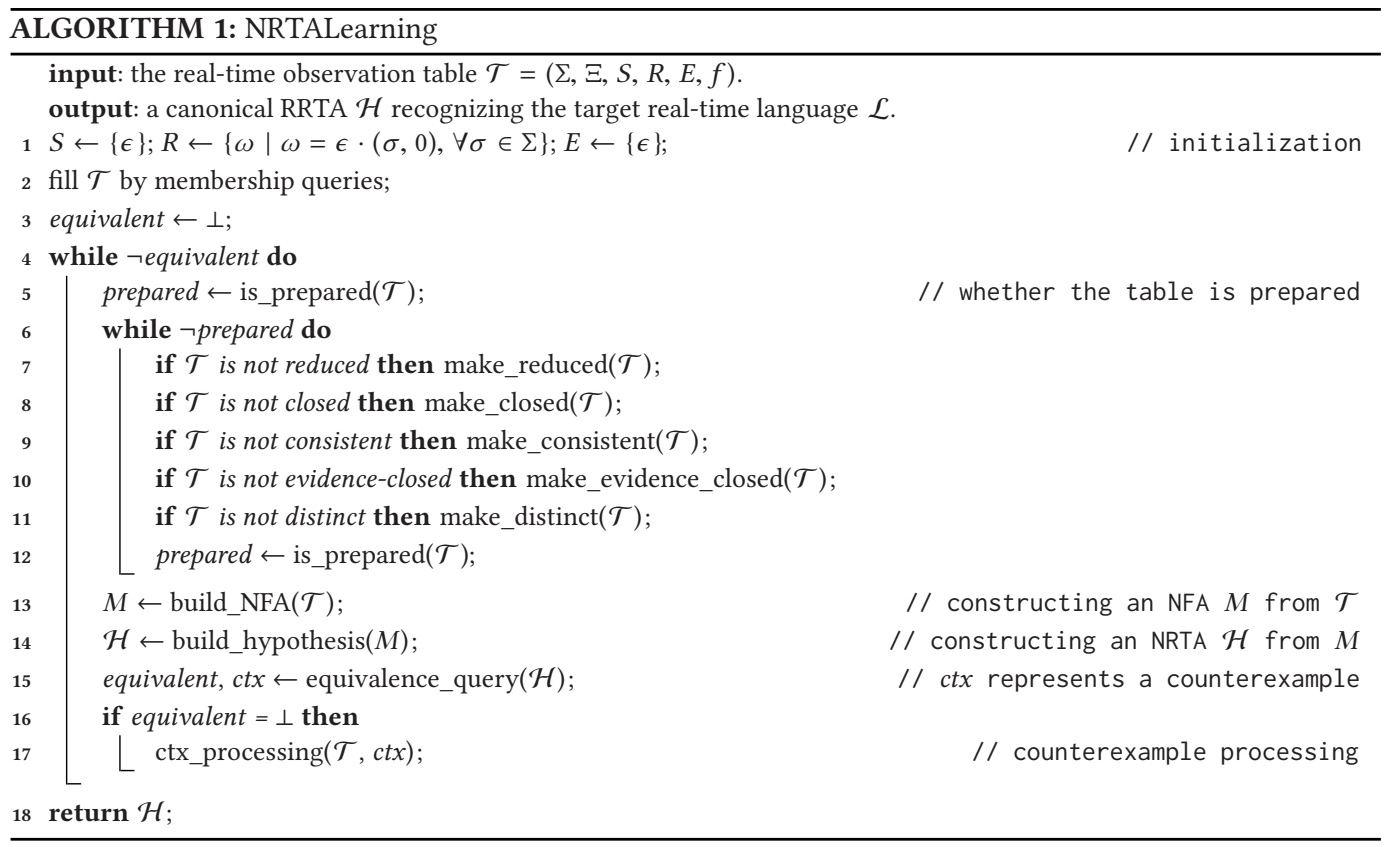

Such a decomposition can be found by using binary search on $\omega^{\prime}$. Then we add all prefixes of $\omega^{\prime}$ to $R$ and all suffixes of $v$ in $E$ instead of adding all prefixes and suffixes of $\omega^{\prime}$ to the table in Step 2. Such operation will reduce the size of $R$ and $E$ comparing to the original Step 2, and thus reduce the number of membership queries. Additionally, it still maintains $S \cup R$ prefix-closed and $E$ suffix-closed.

\subsection{NRTA Learning Algorithm, Correctness and Complexity Analysis}

Algorithm 1 combines all components described in Section 5.1, 5.2 and 5.3. First, the learner makes an initial table $\mathcal{T}$ by adding $\epsilon$ to the prefix set $S$ and the suffix set $E$ by Definition 5.1 and adding $\epsilon \cdot(\sigma, 0)$ to the set of extended prefixes $R$ (Line 1). After filling $\mathcal{T}$ by several membership queries (Line 2), the learner checks if $\mathcal{T}$ is prepared or not (Line 5). If not, repeatedly check each table condition in sequence to find and fix violated one by the operations described in Section 5.1 (Line 711) until the current table $\mathcal{T}$ is prepared. In case $\mathcal{T}$ is prepared, the learner can build an NFA $M$ (Line 13) and a hypothesis NRTA $\mathcal{H}$ afterwards (Line 14) by the construction procedure presented in Section 5.2. Then an equivalence query is performed by submitting $\mathcal{H}$ to the teacher (Line 15). The teacher returns the answer and a counterexample ctx in addition if the answer is negative. After performing a counterexample process (Line 17) described in Section 5.3, the learner needs to check whether the current table $\mathcal{T}$ is prepared again. The whole procedure repeats until the teacher gives a positive answer to an equivalence query, and the algorithm returns the current hypothesis $\mathcal{H}$ as a canonical RRTA recognizing the target real-time language $\mathcal{L}$ (Line 18). The correctness and termination of Algorithm 1 is stated in the following theorem.

Theorem 5.16 (Correctness AND termination). Algorithm 1 terminates and returns the canonical RRTA $\mathcal{H}$ which recognizes the target real-time language $\mathcal{L}$.

Proof. We first show the correctness of Algorithm 1. Suppose Algorithm 1 terminates and return an NRTA $\mathcal{H}$. Since $\mathcal{L}(\mathcal{H})$ passed the equivalence checking with $\mathcal{L}$, then $\mathcal{H}$ recognizes $\mathcal{L}$. Consequently, $\mathcal{H}$ can pass all membership queries on $\omega \cdot e \in(S \cup R) \cdot E$. By Theorem 5.12, the 
NRTA $\mathcal{H}$ is a CRRTA. Then we can conclude that $\mathcal{H}$ is the CRRTA recognizing $\mathcal{L}$. The termination is guaranteed by THEOREM 5.17 proved in the following.

Suppose that the unique minimal DRTA for the target real-time language $\mathcal{L}$ has $n$ locations, the length of the longest returned counterexample is $h$ and let $|\Sigma|=m$. As a convention in active learning, the learning complexity is measured in terms of the number of two kinds of queries rather than the time complexity.

Theorem 5.17 (Complexity). Algorithm 1 takes $\left.O\left(\left(n+n h\left(n^{2}+m \kappa n\right)\right) \cdot h\left(n^{2}+m \kappa n\right)\right)\right)$ membership queries and $O\left(n^{2}+m \kappa n\right)$ equivalence queries to learn the CRRTA recognizing $\mathcal{L}$.

Proof. The proof is similar to that given in [8] for nondeterministic finite automata. We give an outline of the proof below, mainly focusing on the difference from [8]'s.

The main idea is to consider a measure including four natural numbers: $l_{s}$ for the number of distinct rows ever in $S, l$ for the number of distinct rows in the entire table, $p$ for the number of prime rows in the table, and $i$ for the number of strict containing relations of pairs of distinct rows in the table, i.e. $\operatorname{row}(\omega) \subset \operatorname{row}\left(\omega^{\prime}\right)$ where $\omega, \omega^{\prime} \in S \cup R$. Clearly, the value of $l_{s}$, l, and $p$ is upperbounded by $n$. The value of $i$ can only increase when $l$ increases. Whenever $l$ is increased by $k$, the value $i$ increases by at most $k l+k(k-1) / 2$. So $i$ can be increased up to $n(n-1) / 2$.

Next, consider how to measure changes after each operation. If the table is not closed, then extending the table increases $l_{s}$ by 1 and possibly increases $l$ by $k>0$. If the table is not consistent or evidence-closed, extending the table does not change $l_{s}$, and might increase $l$. If $l$ is not increased, then $i$ is decreased by at least 1 . These two cases are exactly the same as for NFA.

Now consider the processing of a counterexample. The main argument in [8] is that if $l$ stays the same, then either $i$ decreases or $p$ increases, for otherwise the automata $\mathcal{H}_{\mathcal{T}}$ and $\mathcal{H}_{\mathcal{T}}$, constructed before and after the processing must be the same, contradicting the fact that $\mathcal{H}_{\mathcal{T}}$, handles an additional counterexample. In our case, there is another possible modification to the automata: splitting an edge into multiple edges by refining the guard. For each distinct row, its guard can be refined by at most $2 \kappa+1$ times, so the total number of refinements is at most $O(m \kappa n)$.

Finally, the process for maintaining the distinctness condition can at most add one row for each distinct row and timed action (pair of an action and a value containing in a region), hence the number of rows added to maintain distinctness is at most $O(m \kappa n)$.

The above argument shows that Algorithm 1 always reaches an equivalence query and terminates after performing $O\left(n^{2}+m \kappa n\right)$ equivalence queries.

Now we consider the number of membership queries. The number of rows in $S$ is upper-bounded by $n$. The operation making $\mathcal{T}$ evidence-closed may add at most $O\left(n \cdot h\left(n^{2}+m \kappa n\right)\right)$ rows in $R$. The counterexample process and the operation for distinctness add at most $O\left(n \cdot h\left(n^{2}+m \kappa n\right)\right)$ rows in $R$ together and $O\left(h\left(n^{2}+m \kappa n\right)\right)$ columns in $E$. Hence, the number of membership queries is at most $\left.O\left(\left(n+n h\left(n^{2}+m \kappa n\right)\right) \cdot h\left(n^{2}+m \kappa n\right)\right)\right)$.

\subsection{Extended- $N L^{*}$}

Suppose $\mathcal{L}$ is a real-time language, based on the corresponding Myhill-Nerode Theorem for realtime languages, there is a constant $\kappa \in \mathbb{N}$ which will be the maximum value appearing in the guards of the corresponding RTAs recognizing $\mathcal{L}$. According to the definition of CRRTAs, assuming that $\kappa$ is known before learning, we can extend $N L^{*}$ algorithm to learn real-time languages as follows.

First construct all regions $[0,0],(0,1), \ldots,[\kappa, \kappa],(\kappa, \infty)$ offline, denoted as $\operatorname{Reg}_{0}, \operatorname{Reg}_{1}, \ldots, \operatorname{Reg}_{2 \kappa+1}$. Then select a number $\tau_{i}^{\prime}$ from each region. If $\operatorname{Reg}_{i}=\left[\tau_{i}, \tau_{i}\right]$ is a point region, then $\tau_{i}^{\prime}=\tau_{i}$. Otherwise, choose $\tau_{i}^{\prime}=\left\lfloor\tau_{i}\right\rfloor+0.1$ where $\tau_{i} \in \operatorname{Reg}_{i}$. After that, an abstract alphabet $\Sigma_{M^{\prime}}=\left\{\left(\sigma, \tau_{i}^{\prime}\right) \mid \sigma \in \Sigma \wedge \tau_{i}^{\prime} \in\right.$ $\left.\operatorname{Reg}_{i} \wedge 0 \leq i \leq 2 \kappa+1\right\}$ can be constructed before learning. $\Sigma_{M^{\prime}}$ is a finite set with $\left|\Sigma_{M^{\prime}}\right|=(2 \kappa+2) \cdot|\Sigma|$. 


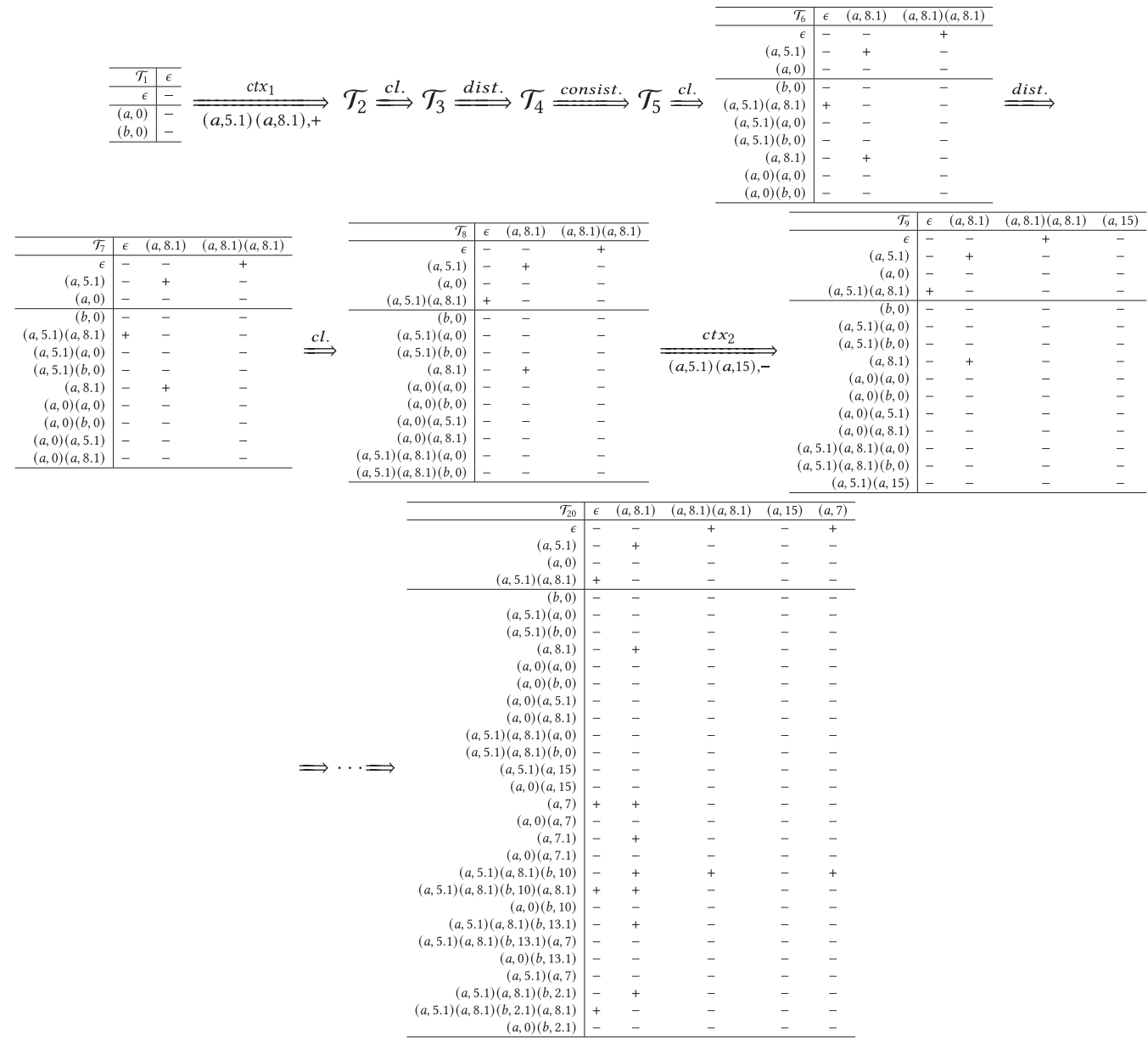

Fig. 6. Some of the table instances generated in the process of learning NRTA $\mathcal{A}$ in Figure 2.

The learning process also follows the two steps construction. At the first step, an NFA $M^{\prime}=$ $\left(Q_{M^{\prime}}, \Sigma_{M^{\prime}}, \Delta_{M^{\prime}}, Q_{M^{\prime}}^{0}, F_{M^{\prime}}\right)$ with the finite alphabet $\Sigma_{M^{\prime}}$ can be constructed using the $N L^{*}$ algorithm directly. At the second step, NFA $M^{\prime}$ is transformed to an NRTA $\mathcal{H}^{\prime}=\left(Q^{\prime}, \Sigma, \Delta^{\prime}, Q_{0}^{\prime}, F^{\prime}\right)$ by reusing the process presented in Section 5.2. From the result in [8], we have the following theorem.

TheOREM 5.18 (ExTENDED-NL*). The Extended-NL* algorithm terminates and returns the CRRTA which recognizes the target real-time language $\mathcal{L}$ after performing $O\left(h \kappa m n^{3}\right)$ membership queries and $O\left(n^{2}\right)$ equivalence queries.

Extended- $N L^{*}$ is a conservative method since it makes membership queries for all regions at all locations. Note that instead of assuming $\kappa$ is known before learning, we can also guess an initial value of $\kappa$, then increase it whenever a counterexample involves a larger time value. However, this may result in restart of the learning process frequently.

\subsection{Running Example}

In this section, we present the learning process for NRTA $\mathcal{A}$ in Figure 2. To simplify the presentation, we omit the evidence-closed condition which has no effect on this example. In Figure 6, $\mathcal{T}_{1}$ is the initial instance of the table. Since $\mathcal{T}_{1}$ is prepared, the learner builds an NFA $M_{1}$ and a 


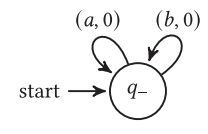

$M_{1}$
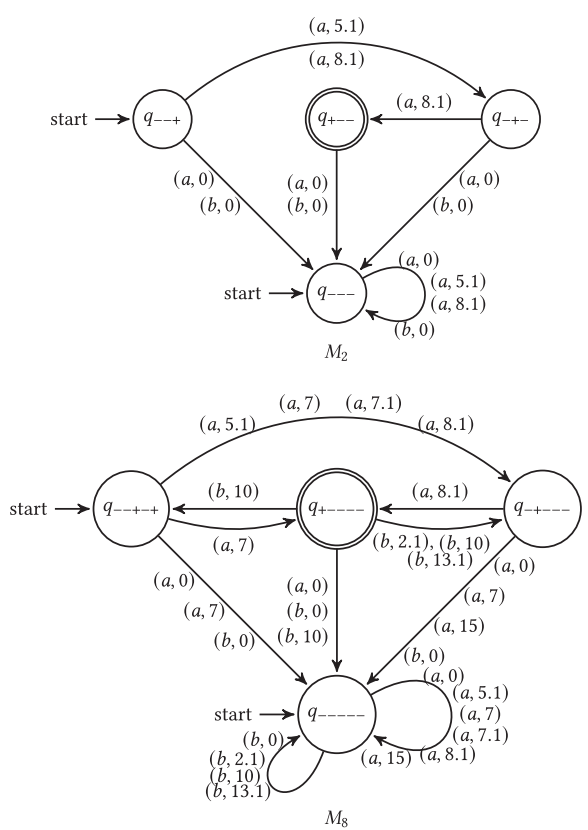

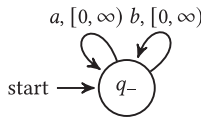

$\mathcal{H}_{1}$
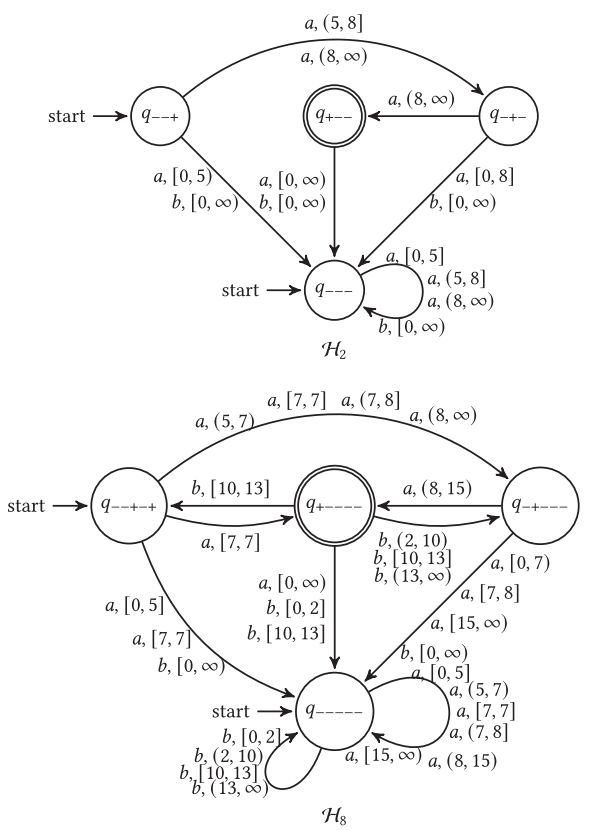

Fig. 7. Some of the NFAs and hypothesis NRTAs generated in the process of learning NRTA $\mathcal{A}$ in Figure 2.

hypothesis NRTA $\mathcal{H}_{1}$ shown in Figure 7 . After making an equivalence query to the teacher, the learner receives a positive counterexample $c t x_{1}=(a, 5.1)(a, 8.1)$. After processing the counterexample, $\mathcal{T}_{2}$ is generated. The procedure from $\mathcal{T}_{2}$ to $\mathcal{T}_{5}$ can be found in Example 5.4. As $\mathcal{T}_{5}$ is not closed, the learner moves $(a, 0)$ from $R$ to $S$. After making the table distinct and closed, the learner obtains another prepared table $\mathcal{T}_{8}$ and builds an NFA $M_{2}$ and hypothesis $\mathcal{H}_{2}$. The learner receives a negative counterexample $c t x_{2}=(a, 5.1)(a, 15)$ and generates $\mathcal{T}_{9}$. Then after 11 more rounds including processing 5 counterexamples, the learner gets a prepared table $\mathcal{T}_{20}$ and builds the final hypothesis $\mathcal{H}_{8}$ which recognizes the target language $\mathcal{L}(\mathcal{A})$. Obviously, if we delete the "sink" location $q_{-----}$ and related transitions, then combine the intervals on the transition from $q_{--+-+}$to $q_{-+---}$and the intervals on the transition from $q_{+----}$to $q_{-+---}$respectively, the resulting automaton will be the same as $\mathcal{A}$ in Figure 2.

\section{EXPERIMENTS}

We implemented the two algorithms NRTALearning and Extended- $N L^{*}$ in Python and evaluated both algorithms on two benchmarks which include a set of randomly generated NRTAs and a set of rational regular expressions. We also demonstrate the algorithm NRTALearning on a scheduling example. All experiments have been conducted on an Intel Core i5-9600 @ 3.1GHz processor with 16GB RAM running 64-bit Debian GNU/Linux 10 equipped with Python 3.7.3.

\subsection{Randomly Generated NRTAs}

In the first experiment, 100 randomly generated NRTAs are divided in 10 groups, with each group having different numbers of locations $|Q|$ and size of alphabet $|\Sigma|$. $|Q|$ ranges from 10 to 20 and $|\Sigma|$ 
Table 1. Experimental Results on the Randomly Generated NRTAs

\begin{tabular}{|c|c|c|c|c|c|c|c|c|c|c|c|}
\hline \multirow{2}{*}{ Group ID } & \multirow{2}{*}{$|\Delta|$} & \multirow{2}{*}{$n_{D R T A}$} & \multirow{2}{*}{ Method } & \multicolumn{3}{|c|}{ \#Membership } & \multicolumn{3}{|c|}{ \#Equivalence } & \multirow{2}{*}{$\left|Q_{\mathcal{H}}\right|$} & \multirow{2}{*}{$t(s)$} \\
\hline & & & & $N_{\min }$ & $N_{\text {mean }}$ & $N_{\max }$ & $N_{\min }$ & $N_{\text {mean }}$ & $N_{\max }$ & & \\
\hline \multirow{3}{*}{ 10_2_20 } & \multirow{3}{*}{23.5} & \multirow{3}{*}{66.5} & NRTA-L & 3520 & 5665.3 & 9660 & 31 & 36.4 & 40 & \multirow{2}{*}{11.1} & 1.7 \\
\hline & & & $\mathrm{E}-N L^{*}$ & 15725 & 26571.5 & 35310 & 3 & 4.7 & 6 & & 1.1 \\
\hline & & & DRTA-L & 38461 & 223977.9 & 670380 & 6 & 9.1 & 13 & 66.5 & 19.3 \\
\hline \multirow{3}{*}{ 10_4_20 } & \multirow{3}{*}{32.3} & \multirow{3}{*}{62.7} & NRTA-L & 4975 & 9664.4 & 15192 & 33 & 49.2 & 62 & \multirow{2}{*}{11.1} & 3.8 \\
\hline & & & $\mathrm{E}-N L^{*}$ & 31433 & 55362.6 & 75618 & 4 & 5.9 & 9 & & 2.3 \\
\hline & & & DRTA-L & 68561 & 347454.2 & 781231 & 7 & 8.7 & 11 & 62.7 & 24.6 \\
\hline \multirow{3}{*}{ 10_6_20 } & \multirow{3}{*}{43.7} & \multirow{3}{*}{79.0} & NRTA-L & 8876 & 17346.3 & 26910 & 52 & 62.8 & 76 & \multirow{2}{*}{11.0} & 10.6 \\
\hline & & & $\mathrm{E}-N L^{*}$ & 68817 & 79628.1 & 96792 & 3 & 5.7 & 8 & & 3.4 \\
\hline & & & DRTA-L & 171886 & 577082.6 & 1234835 & 7 & 8.9 & 11 & 79.0 & 44.1 \\
\hline \multirow{3}{*}{ 10_8_20 } & \multirow{3}{*}{56.5} & \multirow{3}{*}{101.7} & NRTA-L & 14756 & 20309.8 & 29886 & 65 & 83.1 & 97 & \multirow{2}{*}{11.0} & 13.5 \\
\hline & & & $\mathrm{E}-N L^{*}$ & 78642 & 109356.2 & 191550 & 4 & 5.9 & 7 & & 4.6 \\
\hline & & & DRTA-L & 354175 & 1259531.4 & 2848321 & 7 & 9.7 & 13 & 101.7 & 122.3 \\
\hline \multirow{3}{*}{ 10_10_20 } & \multirow{3}{*}{64.1} & \multirow{3}{*}{109.2} & NRTA-L & 12078 & 26558.5 & 56840 & 67 & 92.0 & 118 & & 24.4 \\
\hline & & & $\mathrm{E}-N L^{*}$ & 100816 & 138915.4 & 174746 & 4 & 6.0 & 8 & 11.0 & 6.5 \\
\hline & & & DRTA-L & 807271 & 1632323.2 & 2763231 & 8 & 10.5 & 13 & 109.2 & 176.0 \\
\hline & & & NRTA-L & 8652 & 14968.3 & 21096 & 50 & 66.0 & 80 & & 7.8 \\
\hline 12_4_20 & 42.1 & 127.1 & $\mathrm{E}-N L^{*}$ & 47899 & 74348.9 & 111755 & 4 & 6.0 & 8 & & 3.1 \\
\hline & & & DRTA-L & 88567 & 1071517.5 & 1958611 & 10 & 12.5 & 16 & 127.1 & 117.8 \\
\hline & & & NRTA-L & 10290 & 21305.2 & 38372 & 58 & 73.6 & 85 & & 13.6 \\
\hline 14_4_20 & 49.5 & 130.3 & $\mathrm{E}-N L^{*}$ & 59997 & 101304.0 & 145188 & 4 & 6.7 & 9 & & 4.3 \\
\hline & & & DRTA-L & 422722 & 1014350.4 & 3067064 & 11 & 11.8 & 14 & 130.3 & 97.1 \\
\hline & & & NRTA-L & 18258 & 41004.4 & 132928 & 73 & 85.5 & 103 & & 51.0 \\
\hline 16_4_20 & 56.2 & 325.9 & $\mathrm{E}-N L^{*}$ & 99819 & 128872.7 & 182818 & 3 & 6.2 & 9 & & 5.8 \\
\hline & & & DRTA-L & 408270 & 3883803.1 & 13490655 & 10 & 15.0 & 20 & 325.9 & 909.8 \\
\hline & & & NRTA-L & 14256 & 35404.0 & 61464 & 73 & 97.5 & 128 & & 34.3 \\
\hline 18_4_20 & 61.8 & 330.7 & $\mathrm{E}-N L^{*}$ & 140486 & 178679.7 & 254072 & 7 & 8.6 & 11 & 4 & 7.9 \\
\hline & & & DRTA-L & 613914 & 4989360.0 & 11884279 & 11 & 16.6 & 23 & 330.7 & 899.5 \\
\hline & & & NRTA-L & 26255 & 56817.1 & 135675 & 86 & 104.0 & 117 & & 84.1 \\
\hline 20_4_20 & 68.7 & 422.7 & $\mathrm{E}-N L^{*}$ & 154600 & 211740.0 & 260107 & 6 & 7.6 & 10 & 21.0 & 9.9 \\
\hline & & & DRTA-L & 2921594 & 6720561.6 & 13160576 & 17 & 18.6 & 22 & 422.7 & 1392.3 \\
\hline
\end{tabular}

Group ID: each group has ID of the form $|Q|_{-}|\Sigma|_{-} \kappa$, where $|Q|$ is the number of locations, $|\Sigma|$ is the size of the alphabet, and $\kappa$ is the maximum constant appearing in the clock constraints.

$|\Delta|$ : average number of transitions of an NRTA in the corresponding group.

$n_{D R T A}$ : average number of locations of the corresponding minimal DRTAs for each group.

Method: NRTA-L, E- $N L^{*}$, and DRTA-L representing the learning algorithms NRTALearning, Extended- $N L^{*}$ and the learning algorithm for DRTAs in [5], respectively.

\#Membership \& \#Equivalence: number of membership and equivalence queries, respectively. $N_{\min }$ : minimal,

$N_{\text {mean }}$ : mean, $N_{\text {max }}$ : maximum.

$\left|Q_{\mathcal{H}}\right|$ : average number of locations of the learned automata for each group.

$t$ : average wall-clock time in seconds, including both time taken by the learner and time taken by the teacher.

ranges from 2 to 10 . We set the maximum constant appearing in guards $\kappa=20$. We abbreviate the algorithms NRTALearning and Extended- $N L^{*}$ as NRTA-L and E- $N L^{*}$ respectively. DRTA-L denotes the learning algorithm for DRTAs in [5]. The experimental results are shown in Table 1.

The three algorithms are all successful to learn out all 100 models. For all groups, NRTA-L takes fewer membership queries than $E-N L^{*}$ and DRTA-L takes the most number of membership queries to learn the corresponding minimal DRTAs. NRTA-L is more radical for guessing the bounds of guards by using the partition function while $\mathrm{E}-N L^{*}$ is more conservative. However, the drawback of the radical operation is that we need more equivalence queries to correct the guards, which explains why NRTA-L takes more equivalence queries. Since the language equivalence problem 

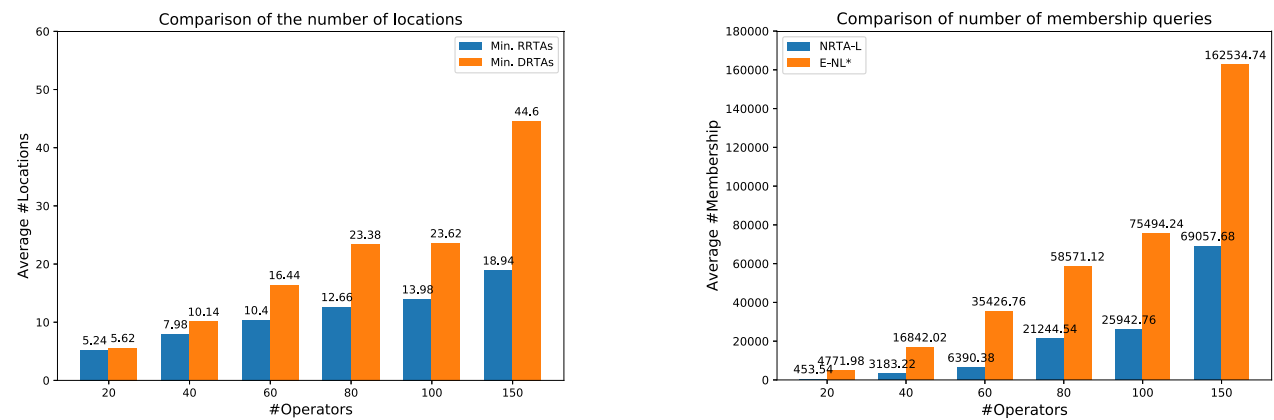

Fig. 8. Comparison results on randomly generated rational regular expressions. The expressions are divided into 6 groups according to the number of the used operators (\#Operators). Left: Comparison of the average number of locations between the learnt minimal RRTAs and the corresponding minimal DRTAs. Right: Comparison of the average number of membership queries performed by NRTA-L and E-NL*.

of NRTAs is PSPACE-complete, more equivalence queries means more average running time for NRTA-L. Note that the complexity is measured in terms of the number of queries in the automaton learning theory as we mentioned before. The other observation is that the learnt CRRTAs are much smaller (w.r.t. the number of locations) than the corresponding minimal DRTAs, which conforms to the contents on residual real-time languages in Section 4.

\subsection{Randomly Generated Rational Regular Expressions}

In the second experiment, following [13], we fixed a set of operators $\{\emptyset, \Sigma, *, \cdot,+\}$, where $\Sigma=$ $\{a, b, c\}$ and $*, \cdot,+$ represent the Kleene star, concatenation and union respectively. Then we randomly generated 300 different rational regular expressions based on the operators. These real-time languages are divided into 6 groups according to the number of operators used. The distribution $p$ on operators used for random generation is $p_{\epsilon}=0.02, p_{\Sigma}=0.1, p_{*}=0.13, p .=0.5$ and $p_{+}=0.25$. Both NRTA-L and E-NL* are successful in learning all real-time languages. In Figure 8 , the chart on the left shows that the learnt RRTAs are much smaller than the corresponding minimal DRTAs. The chart on the right shows NRTA-L takes fewer membership queries than $E-N L^{*}$ on average.

\subsection{A Scheduling Example}

For the third experiment, we follow a case study conducted in [6] (as Experiment 6.2). It considers the scheduling of final testing of integrated circuits. We first review the setting of the case study in that paper. There are two kinds of products $A$ and $B$ which are processed in two stages: testing and burn_in. The two jobs for $A$ and $B$ are grouped into 5 and 2 lots respectively. In the testing stage, the lots are processed serially on two parallel machines, and it takes one machine 3 time units to handle each $A$ lot and 4 time units to handle each $B$ lot. In the burn_in stage, all lots are processed in a batch manner on the two machines, and it takes one machine 10 time units to finish each batch. The maximum batch size for one machine is 5 . During testing, if a machine is allocated to process $A$ products, it cannot handle $B$ products until all $A$ jobs are finished. Likewise for machines that are allocated to process $B$ products. We assume that it takes $t \in(0,1]$ time to prepare one machine and $t \in[1,2]$ time to prepare two machines in each stage. The operations in one stage can start only after both machines are allocated and prepared. We also assume that products are sent to the burn_in stage only after the testing stage is completely finished.

The schedules for processing $A$ and $B$ products are modeled by NRTAs $M_{A}$ and $M_{B}$ (see Figure $9(\mathrm{a})$ and $9(\mathrm{~b})$ ), respectively. $M_{A}$ has nine locations $0, \ldots, 8$. Here 0 is the initial location, 1 


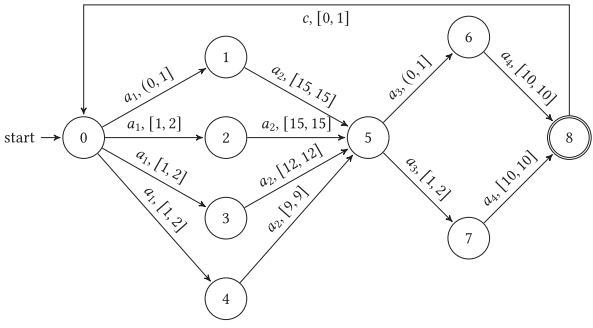

(a) RTA $M_{A}$ for the schedule of product $A$

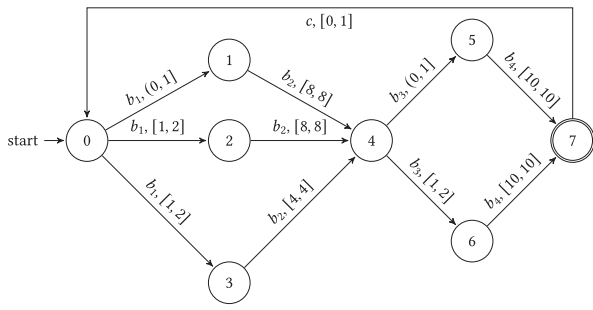

(b) RTA $M_{B}$ for the schedule of product $B$

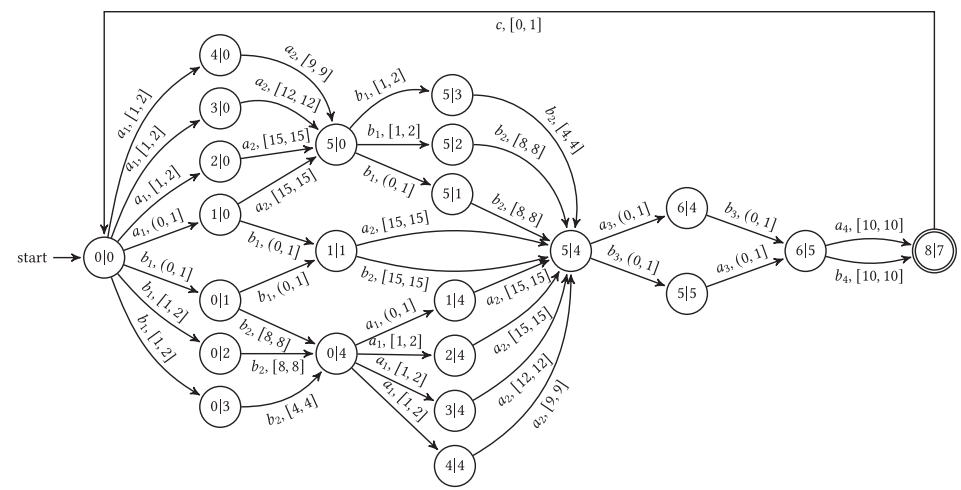

(c) NRTA $M_{A B}$ for the composed schedule of two products $A$ and $B$. The locations are in the form of $q_{A} \mid q_{B}$, where $q_{A}\left(q_{B}\right)$ represents the corresponding location of $M_{A}\left(M_{B}\right)$.

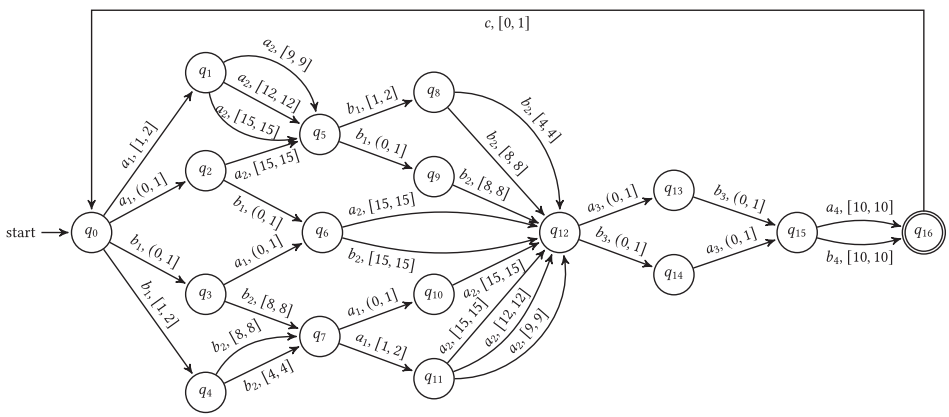

(d) The learnt NRTA $\mathcal{H}$ excluding the "sink" location and relevant transitions

Fig. 9. (a) NRTA $M_{A}$; (b) NRTA $M_{B}$; (c) The composed NRTA $M_{A B}$; (d) The learnt NRTA $\mathcal{H}$.

for allocating one machine to test $A$ products, and 2, 3, 4 for allocating two machines, but with different scheduling policies. In 2 , one machine has 5 lots and the other has no lots; in 3 , one machine has 4 lots and the other has 1 lot; in 4, one machine has 3 lots and the other has 2 lots. Location 5 indicates the testing stage has finished. Locations 6, 7 stand for allocating $A$ lots for burn_in on one machine and two machines respectively. The accepting location 8 stands for completion of processing, with return to initial location after a delay in [0,1]. The labels $a_{1}, a_{2}, a_{3}, a_{4}$ and $c$ represent allocating machines for testing, testing finished, allocating machines for burn_in, burn_in finished, and completion, respectively. The NRTA $M_{B}$ with the labels $b_{1}, b_{2}, b_{3}$ and $b_{4}$ can be understood similarly. 
Assuming that we always allocate one machine for each kind of product during the second stage, the two schedules can be composed to form a schedule model for two products, given by an NRTA $M_{A B}$ with 23 locations connected by 39 transitions. The composed model is shown in Figure 9(c). Using the method NRTA-L, the CRRTA $\mathcal{H}$ (see Figure 9(d)) for the composed model is learned in 12.4 seconds after 10670 membership queries and 50 equivalence queries. The resulting $\mathcal{H}$ is equivalent to $M_{A B}$ but simpler, in particular it has only 17 locations excluding the sink location, connected by 33 transitions.

\section{CONCLUSION AND DISCUSSION}

In this paper, we presented two active learning algorithms for NRTAs. One is in the standard MAT framework, and the other is with additionally assuming that the maximum constant appearing in the clock constraints is known before learning. Before that, we proved a corresponding MyhillNerode theorem for real-time languages which shows that there exists a unique minimal DRTA for a given real-time language but it does not hold for NRTAs. In order to set a learning target, we defined residual real-time automata and proved that for a real-time language there is a unique minimal RRTA recognizing it. As a result, learning NRTAs is transformed to learn RRTAs.

As mentioned before, RTAs are a kind of one-clock timed automata which reset the unique clock at every transition. Hence, it is restricted in that it cannot represent the common timing constraints involving multiple locations and actions. However, RTAs are still powerful enough for specifying the functionality of key-distribution protocols and modelling scheduling problems in practice. We leave for future work to adapt our algorithm to learn nondeterministic one-clock automata whose expressiveness is strictly stronger than deterministic ones and RTAs. For learning general timed automata, one way is to construct the corresponding region automata. However, it brings an exponential explosion on the size of model. How to learn a succinct model still needs more work.

\section{REFERENCES}

[1] Bernhard K. Aichernig, Andrea Pferscher, and Martin Tappler. 2020. From passive to active: Learning timed automata efficiently. In Proceedings of the 12th International Symposium NASA Formal Methods, NFM 2020 (LNCS, Vol. 12229). Springer, 1-19. https://doi.org/10.1007/978-3-030-55754-6_1

[2] Rajeev Alur and David L. Dill. 1994. A theory of timed automata. Theoretical Computer Science 126, 2 (1994), $183-235$. https://doi.org/10.1016/0304-3975(94)90010-8

[3] Rajeev Alur, P. Madhusudan, and Wonhong Nam. 2005. Symbolic compositional verification by learning assumptions. In Proceedings of the 17th International Conference on Computer Aided Verification, CAV 2005 (LNCS, Vol. 3576). Springer, Heidelberg, 548-562. https://doi.org/10.1007/11513988_52

[4] Jie An, Mingshuai Chen, Bohua Zhan, Naijun Zhan, and Miaomiao Zhang. 2020. Learning one-clock timed automata. In Proceedings of the 26th International Conference on Tools and Algorithms for the Construction and Analysis of Systems, TACAS 2020 (LNCS, Vol. 12078). Springer, Heidelberg, 444-462. https://doi.org/10.1007/978-3-030-45190-5_25

[5] Jie An, Lingtai Wang, Bohua Zhan, Naijun Zhan, and Miaomiao Zhang. 2020. Learning real-time automata. SCIENCE CHINA Information Sciences (2020). https://www.sciengine.com/doi/10.1007/s11432-019-2767-4. In press.

[6] Jie An, Naijun Zhan, Xiaoshan Li, Miaomiao Zhang, and Wang Yi. 2018. Model checking bounded continuous-time extended linear duration invariants. In Proceedings of the 21st International Conference on Hybrid Systems: Computation and Control (part of CPS Week), HSCC 2018. ACM, 81-90. https://doi.org/10.1145/3178126.3178147

[7] Dana Angluin. 1987. Learning regular sets from queries and counterexamples. Information and Computation 75, 2 (1987), 87-106. https://doi.org/10.1016/0890-5401(87)90052-6

[8] Benedikt Bollig, Peter Habermehl, Carsten Kern, and Martin Leucker. 2009. Angluin-style learning of NFA. In Proceedings of the 21st International foint Conference on Artificial Intelligence, IfCAI 2009. 1004-1009.

[9] Filippo Bonchi and Damien Pous. 2013. Checking NFA equivalence with bisimulations up to congruence. In Proceedings of the 40th Annual ACM SIGPLAN-SIGACT Symposium on Principles of Programming Languages, POPL 2013. ACM, 457-468. https://doi.org/10.1145/2429069.2429124 
[10] Ben Caldwell, Rachel Cardell-Oliver, and Tim French. 2016. Learning time delay mealy machines from programmable logic controllers. IEEE Trans Autom. Sci. Eng. 13, 2 (2016), 1155-1164. https://doi.org/10.1109/TASE.2015.2496242

[11] Colin de la Higuera. 2010. Grammatical Inference: Learning Automata and Grammars. Cambridge University Press.

[12] François Denis, Aurélien Lemay, and Alain Terlutte. 2001. Residual finite state automata. In Proceedings of the 18th Annual Symposium on Theoretical Aspects of Computer Science, STACS 2001 (LNCS, Vol. 2010). Springer, Heidelberg, 144-157. https://doi.org/10.1007/3-540-44693-1_13

[13] François Denis, Aurélien Lemay, and Alain Terlutte. 2004. Learning regular languages using RFSAs. Theoretical Computer Science 313, 2 (2004), 267-294. https://doi.org/10.1016/j.tcs.2003.11.008

[14] Dorothy E. Denning and Giovanni Maria Sacco. 1981. Timestamps in key distribution protocols. Commun. ACM 24, 8 (1981), 533-536. https://doi.org/10.1145/358722.358740

[15] Catalin Dima. 2001. Real-time automata. Journal of Automata, Languages and Combinatorics 6, 1 (2001), 3-23. https: //doi.org/10.25596/jalc-2001-003

[16] Samuel Drews and Loris D’Antoni. 2017. Learning symbolic automata. In Proceedings of the 23rd International Conference on Tools and Algorithms for the Construction and Analysis of Systems, TACAS 2017 (LNCS, Vol. 10205). Springer, Heidelberg, 173-189. https://doi.org/10.1007/978-3-662-54577-5_10

[17] Paul Fiterau-Brostean, Ramon Janssen, and Frits W. Vaandrager. 2016. Combining model learning and model checking to analyze TCP implementations. In Proceedings of the 28th International Conference on Computer Aided Verification, CAV 2016 (LNCS, Vol. 9780). Springer, Heidelberg, 454-471. https://doi.org/10.1007/978-3-319-41540-6_25

[18] Olga Grinchtein, Bengt Jonsson, and Martin Leucker. 2010. Learning of event-recording automata. Theoretical Computer Science 411, 47 (2010), 4029-4054. https://doi.org/10.1016/j.tcs.2010.07.008

[19] Léo Henry, Thierry Jéron, and Nicolas Markey. 2020. Active learning of timed automata with unobservable resets. In Proceedings of the 18th International Conference on Formal Modeling and Analysis of Timed Systems, FORMATS 2020 (LNCS, Vol. 12288). Springer, 144-160. https://doi.org/10.1007/978-3-030-57628-8_9

[20] Xiangyu Jin, Jie An, Bohua Zhan, Naijun Zhan, and Miaomiao Zhang. 2021. Inferring nonlinear switched dynamical systems. Formal Aspects of Computing (2021). https://doi.org/10.1007/s00165-021-00542-7

[21] Oded Maler and Amir Pnueli. 1995. On the learnability of infinitary regular sets. Information and Computation 118, 2 (1995), 316-326. https://doi.org/10.1006/inco.1995.1070

[22] Ronald L. Rivest and Robert E. Schapire. 1993. Inference of finite automata using homing sequences. Information and Computation 103, 2 (1993), 299-347. https://doi.org/10.1006/inco.1993.1021

[23] Jana Schmidt, Asghar Ghorbani, Andreas Hapfelmeier, and Stefan Kramer. 2013. Learning probabilistic real-time automata from multi-attribute event logs. Intelligent Data Analysis 17, 1 (2013), 93-123. https://doi.org/10.3233/IDA120569

[24] Miriam García Soto, Thomas A. Henzinger, Christian Schilling, and Luka Zeleznik. 2019. Membership-based synthesis of linear hybrid automata. In Proceedings of the 31st International Conference on Computer Aided Verification, CAV 2019 (LNCS, Vol. 11561). Springer, Heidelberg, 297-314. https://doi.org/10.1007/978-3-030-25540-4_16

[25] Martin Stigge, Pontus Ekberg, Nan Guan, and Wang Yi. 2011. The digraph real-time task model. In Proceedings of the 17th IEEE Real-Time and Embedded Technology and Applications Symposium, RTAS 2011. IEEE Computer Society, 71-80. https://doi.org/10.1109/RTAS.2011.15

[26] Martin Tappler, Bernhard K. Aichernig, Kim Guldstrand Larsen, and Florian Lorber. 2019. Time to learn - learning timed automata from tests. In Proceedings of the 17th International Conference on Formal Modeling and Analysis of Timed Systems, FORMATS 2019 (LNCS, Vol. 11750). Springer, 216-235. https://doi.org/10.1007/978-3-030-29662-9_13

[27] Frits W. Vaandrager. 2017. Model learning. Commun. ACM 60, 2 (2017), 86-95. https://doi.org/10.1145/2967606

[28] Frits W. Vaandrager, Roderick Bloem, and Masoud Ebrahimi. 2021. Learning mealy machines with one timer. In Proceedings of the 15th International Conference on Language and Automata Theory and Applications, LATA 2021 (LNCS, Vol. 12638). Springer, 157-170. https://doi.org/10.1007/978-3-030-68195-1_13

[29] Sicco Verwer. 2010. Efficient Identification of Timed Automata: Theory and practice. Ph.D. Dissertation. Delft University of Technology, Netherlands.

[30] Sicco Verwer, Mathijs de Weerdt, and Cees Witteveen. 2009. One-clock deterministic timed automata are efficiently identifiable in the limit. In Proceedings of the 3rd International Conference on Language and Automata Theory and Applications, LATA 2009 (LNCS, Vol. 5457). Springer, 740-751. https://doi.org/10.1007/978-3-642-00982-2_63

[31] Sicco Verwer, Mathijs de Weerdt, and Cees Witteveen. 2011. The efficiency of identifying timed automata and the power of clocks. Information and Computation 209, 3 (2011), 606-625. https://doi.org/10.1016/j.ic.2010.11.023

[32] Sicco Verwer, Mathijs de Weerdt, and Cees Witteveen. 2012. Efficiently identifying deterministic real-time automata from labeled data. Machine Learning 86, 3 (2012), 295-333. https://doi.org/10.1007/s10994-011-5265-4

[33] Sicco Verwer, Mathijs De Weerdt, and Cees Witteveen. 2007. An algorithm for learning real-time automata. Electrical Engineering Mathematics \& Computer Science (2007). 
[34] Gail Weiss, Yoav Goldberg, and Eran Yahav. 2018. Extracting automata from recurrent neural networks using queries and counterexamples. In Proceedings of the 35th International Conference on Machine Learning, ICML 2018 (PMLR, Vol. 80). PMLR, 5244-5253.

[35] Gail Weiss, Yoav Goldberg, and Eran Yahav. 2019. Learning deterministic weighted automata with queries and counterexamples. In Proceedings of the 33rd Annual Conference on Neural Information Processing Systems 2019, NeurIPS 2019. 8558-8569.

Received April 2021; revised June 2021; accepted July 2021 